\title{
Observations of the Dispersion Characteristics and Meridional Sea Level Structure of Equatorial Waves in the Pacific Ocean
}

\author{
J. THOMAS FARRAR \\ Department of Physical Oceanography, Woods Hole Oceanographic Institution, Woods Hole, Massachusetts
}

(Manuscript received 3 August 2007, in final form 12 December 2007)

\begin{abstract}
Spectral techniques applied to altimetry data are used to examine the dispersion relation and meridional sea level structure of wavelike variability with periods of about 20 to 200 days in the equatorial Pacific Ocean. Zonal wavenumber-frequency power spectra of sea surface height, when averaged over about $7^{\circ} \mathrm{S}-7^{\circ} \mathrm{N}$, exhibit spectral peaks near the theoretical dispersion curves of first baroclinic-mode equatorial Kelvin and Rossby waves. There are distinct, statistically significant ridges of power near the first and second meridional-mode Rossby wave dispersion curves. Sea level variability near the theoretical Kelvin wave and first meridional-mode Rossby wave dispersion curves is dominantly (but not perfectly) symmetric about the equator, while variability near the theoretical second meridional-mode Rossby wave dispersion curve is dominantly antisymmetric. These results are qualitatively consistent with expectations from classical or shear-modified theories of equatorial waves.

The meridional structures of these modes resemble the meridional modes of equatorial wave theory, but there are some robust features of the meridional profiles that were not anticipated. The meridional sea level structure in the intraseasonal Kelvin wave band closely resembles the theoretically expected Gaussian profile, but sea level variability coherent with that at the equator is detected as far away as $11.75^{\circ} \mathrm{S}$, possibly as a result of the forced nature of these Kelvin waves. Both first and second meridional-mode Rossby waves have larger amplitude in the Northern Hemisphere. The meridional sea level structure of tropical instability waves closely resembles that predicted by Lyman et al. using a model linearized about a realistic equatorial zonal current system.
\end{abstract}

\section{Introduction}

For at least 40 years, researchers have fully appreciated that the vanishing of the Coriolis "force" at the equator causes the near-equatorial regions to act as a waveguide for atmospheric and oceanic waves of frequencies low enough to be affected by the earth's rotation (e.g., Blandford 1966; Matsuno 1966; Munk and Moore 1968). Waves in the equatorial waveguide tend to propagate zonally and are organized into a set of discrete meridional modes. There is now a vast body of literature providing observational confirmation, at least qualitatively, of predictions about these waves from linear, shallow-water theory on the $-\beta$ plane (Matsuno 1966; Munk and Moore 1968) in both the atmosphere (e.g., Wallace and Kousky 1968; Zangvil and Yanai

Corresponding author address: J. Thomas Farrar, Woods Hole Oceanographic Institution, Mail Stop 29, Woods Hole, MA 02543. E-mail: jfarrar@whoi.edu
1980; Takayabu 1994; Wheeler and Kiladis 1999; Roundy and Frank 2004a) and the ocean (e.g., Wunsch and Gill 1976; Ripa and Hayes 1981; Eriksen and Richman 1988; Delcroix et al. 1991; Chelton et al. 2003).

The most striking demonstration of equatorial waves obeying approximately the dispersion relationships of classical equatorial wave theory is found in the meteorological literature (Takayabu 1994; Wheeler and Kiladis 1999; Roundy and Frank 2004). Using satellite observations of outgoing longwave radiation, Wheeler and Kiladis (1999) and Roundy and Frank (2004a) estimated zonal wavenumber-frequency spectra averaged over the "equatorial belt," the latitudinal range where equatorial dynamics are expected to be applicable. These spectra show distributions of power that clearly resemble the dispersion curves of classical theory. Averaging the spectra over a range of latitudes near the equator sidesteps one of the chief difficulties in identifying modes of variability resembling the meridional modes of classical theory: the dynamical fields of the various modes have maxima that occur at different lati- 
tudes. For example, the peak signal in pressure for a Kelvin wave occurs at the equator, the peak pressure signal of the gravest meridional-mode Rossby wave occurs roughly two deformation radii off of the equator, and the peak pressure signal of the second meridionalmode Rossby wave occurs slightly farther poleward. Thus, when considering zonal wavenumber-frequency spectra at a single latitude, only certain modes can be expected to be apparent, but when the spectra from a number of latitudes within the equatorial belt are averaged, contributions from a number of modes may be seen at once.

Progress in isolating and understanding the dispersion characteristics of equatorial waves in the atmosphere has also been made by using a simple procedure for decomposing data into fields that are symmetric or antisymmetric about the equator (Yanai and Murakami 1970; Zangvil and Yanai 1980). This filtering procedure allows one to capitalize on theoretical expectations about the symmetry properties of the various equatorial waves, and it has proven useful for isolating the equatorial wave modes, even when the modes do not have the perfectly symmetric or antisymmetric meridional structures predicted by classical theory (Roundy and Frank 2004a). Wavenumber-frequency spectra of symmetric and antisymmetric filtered outgoing longwave radiation clearly reveal the equatorially trapped Kelvin, Rossby, gravity, and mixed Rossby-gravity waves expected from classical theory (Wheeler and Kiladis 1999; Roundy and Frank 2004a).

There have been few studies examining the characteristics of oceanic equatorial waves in the zonal wavenumber-frequency domain, despite the utility of such a spectral approach for gaining insight into the dispersion characteristics of equatorial waves. Historically, spatial and temporal sparsity of data has made such analyses difficult (e.g., Eriksen 1982; Eriksen and Richman 1988), but satellite-based altimetric observations during the last couple of decades provide the broad, continuous spatial and temporal coverage needed for examination of the characteristics of equatorial waves in the wavenumber-frequency domain. [The Tropical Atmosphere Ocean (TAO)-Triangle Trans-Ocean Buoy Network (TRITON) (McPhaden et al. 1998) in the tropical Pacific would also allow such analyses, but those data will not be used here.] Studies using satellite altimetry observations to characterize the variability of equatorial sea level as a function of both zonal wavenumber and frequency are rare (e.g., Périgaud 1990; Zang et al. 2002; Wakata 2007). The present study extends these previous efforts by applying methods used in the meteorological literature (Yanai and Murakami 1970; Zangvil and Yanai 1980; Wheeler and Kiladis
1999; Roundy and Frank 2004a) to altimetric data so as to allow further insight into the spectral characteristics of equatorial waves in the Pacific Ocean at periods exceeding a few weeks. As will be shown, this approach allows isolation of the Kelvin wave mode and at least two distinct equatorial Rossby wave modes.

There have also been relatively few studies using observations to characterize the meridional structure of equatorially trapped wave modes in the ocean. Wunsch and Gill (1976) pioneered these efforts using tide-gauge data from a handful of island stations in the Pacific, successfully isolating some equatorially trapped gravity wave modes and examining their meridional structure to the extent allowed by the scattered data records. The meridional structure of equatorial Kelvin waves has been examined using in situ observations (Ripa and Hayes 1981; Johnson and McPhaden 1993) and satellite altimetry (Delcroix et al. 1991; Cravatte et al. 2003). The meridional structure of mixed Rossby-gravity waves has been examined using measurements of subsurface temperature (Lyman et al. 2007) and satellite sea surface temperature (Tsai et al. 1992). The meridional structure of equatorial Rossby waves has been examined (mostly at the annual period and exclusively for the first meridional mode) in subsurface temperature (Lukas and Firing 1985; Kessler and McCreary 1993) and in satellite altimetry (Delcroix et al. 1991; Chelton et al. 2003; Perez et al. 2005). Finally, meridionally coherent variations associated with tropical instability waves have also been studied using in situ and satellite observations (e.g., McPhaden 1996; Chelton et al. 2000; Lyman et al. 2005). The present study complements these earlier studies by using a novel approach to extract the meridional sea level structure in particular wavenumber-frequency bands, allowing some aspects of the meridional structure of these wavelike motions to be examined at a level of detail not seen in previous studies.

This paper examines the dispersion relation and meridional sea level structure of equatorial Kelvin waves, first and second meridional-mode Rossby waves, and tropical instability waves in the Pacific Ocean and is organized as follows. Section 2 describes the data and analysis methods; section 3 presents some theoretical background that facilitates understanding of the methods and results; section 4 describes the primary results involving zonal wavenumber-frequency spectra of sea level; section 5 presents estimates of the meridional structure of some of the modes of variability identified in section 4 and discusses these structures in the context of previous work; and conclusions are presented in section 6. 


\section{Data and methods}

The sea surface height ( $\mathrm{SSH}$ ) anomaly data used here come from the version $3,0.5^{\circ}$ (along-track binned), 10 day binned Ocean Topography Experiment (TOPEX)/ Poseidon SSH anomaly dataset distributed in association with the World Ocean Circulation Experiment (WOCE). These SSH anomaly data and details regarding their processing can be obtained from the NASA Jet Propulsion Laboratory's Physical Oceanography Distributed Active Archive Center (http://podaacwww.jpl.nasa.gov/DATA_PRODUCT/OCC/; the dataset is JPL-PO.DAAC Product 172).

The data are bin averaged onto a global $0.5^{\circ}$ by $0.5^{\circ}$ grid, but only the grid cells through which the TOPEX/ Poseidon ground track passes contain data. This gridding procedure produces a field that has zonal spacings as small as $0.5^{\circ}$ and as large as $3^{\circ}$, and the bins for which data are reported vary between repeat cycles of the satellite. Prior to analysis, the data were linearly interpolated (in longitude) to a $0.5^{\circ}$ grid. This interpolation of measurements from nonuniformly spaced longitudes leads to a poorly defined Nyquist wavelength, but a conservative estimate of the effective Nyquist wavelength is $6^{\circ}$. Attention is restricted here to variability with zonal wavelengths greater than $12^{\circ}$, which is well resolved in the spectral calculations, so the results should be insensitive to the details of interpolation.

The principal methods used involve computing zonal wavenumber-frequency autospectra of SSH with and without application of a filter designed to isolate variability that is symmetric or antisymmetric about the equator. Other methods used involve computation of cross-spectra between longitude-time sections of SSH at different latitudes and subsequent estimation of the coherence amplitude, coherence phase, and gain. For all of these calculations, SSH data span the time period from 1993 to 2001 and longitudes from $148.75^{\circ} \mathrm{E}$ to $87.75^{\circ} \mathrm{W}$. Data gaps caused by the presence of islands were filled by linear interpolation in longitude.

This study examines the zonal wavenumberfrequency power spectra of SSH variability that is symmetric or antisymmetric about the equator using a filtering procedure that has been used to examine equatorial waves in the atmosphere (Yanai and Murakami 1970; Zangvil and Yanai 1980; Wheeler and Kiladis 1999; Roundy and Frank 2004a) and the ocean (Tsai et al. 1992; Meyers et al. 1993; Meyers and O'Brien 1994). This approach is based on the fact that any field, even an asymmetric one, can be decomposed into symmetric and antisymmetric contributions by adding or subtracting the signal from opposite locations about the axis of symmetry. It is easy to see how this procedure acts as a symmetry filter: by adding (or subtracting) the signal at opposite latitudes, the equatorially antisymmetric (or symmetric) contribution is cancelled out.

The symmetric (antisymmetric) contribution to the $\mathrm{SSH}$ in the equatorial region is constructed by adding (subtracting) the signal at each latitude on opposite sides of the equator and dividing by a normalization constant. The normalization constant is chosen to be $\sqrt{2}$ so that the average of the symmetric and antisymmetric SSH spectra is equal to the total SSH spectrum averaged over the same latitudes. (Averaging of the spectra from different latitudes is described below.) It is widely appreciated that equatorial wave modes can be asymmetric (e.g., Chelton et al. 2003; Perez et al. 2005; Lyman et al. 2005; see references therein for earlier evidence of cross-equatorial asymmetry). Other approaches, described below, are used to examine asymmetries.

The zonal wavenumber-frequency spectrum of a given longitude-time section of SSH (or sum or difference of SSH at opposing latitudes) was estimated by a method similar to that described in Farrar and Weller (2006) using a two-dimensional fast Fourier transform (2DFFT). The longitude-time mean was removed from each longitude-time section before tapering the edges of each section to zero with a half-Hanning window over 220 days and $10^{\circ}$ longitude. Spectra were not corrected for energy loss associated with the tapering. Periodograms were produced by computing the squared 2DFFT of the entire record.

The 2D periodograms from different latitudes, assumed to have uncorrelated noise, were averaged to improve the statistical stability of the spectral estimate. The fact that the peak amplitudes of the various equatorial meridional modes are expected to occur at different latitudes also provides physical motivation for averaging spectral estimates from different latitudes over a range of latitudes near the equator. For the symmetric/antisymmetric filtered fields, periodograms constructed from the sums or differences of latitude pairs from $0.25^{\circ} \mathrm{N}$ and $\mathrm{S}$ to $6.75^{\circ} \mathrm{N}$ and $\mathrm{S}$ were averaged. For unfiltered SSH variability, averages over the Northern Hemisphere $\left(0.25^{\circ}-6.75^{\circ} \mathrm{N}\right)$, Southern Hemisphere $\left(0.25^{\circ}-6.75^{\circ} \mathrm{S}\right)$, and equatorial belt $\left(6.75^{\circ} \mathrm{S}-6.75^{\circ} \mathrm{N}\right)$ were examined. Examining the SSH spectra from the Northern Hemisphere and Southern Hemisphere separately gives some information relevant to the asymmetry of equatorial variability.

After averaging periodograms from different latitudes, nonoverlapping averaging was carried out across seven adjacent frequency bands. To retain the best possible wavenumber resolution, no wavenumber band averaging was performed. For the $6.75^{\circ} \mathrm{S}-6.75^{\circ} \mathrm{N}$ equato- 
rial belt, this averaging scheme gives 392 degrees of freedom $(28$ records $\times 7$ bands averaged $\times 2$ degrees of freedom from the FFT). For the Northern Hemisphere, Southern Hemisphere, symmetric, and antisymmetric spectra, there are 192 degrees of freedom, half as many as for the full $6.75^{\circ} \mathrm{S}-6.75^{\circ} \mathrm{N}$ spectrum. Confidence intervals $(95 \%$ and $99 \%)$ for the spectra were computed as described by Bendat and Piersol (1986, p. 286, Eq. 8.159). The bandwidth (or spectral resolution) for the power spectra is about $1 / 124^{\circ}$ by $1 /(469.3$ days).

In section 5, the meridional structure of variability in selected wavenumber-frequency bands associated with particular types of waves will be examined. This is done by computing the coherence amplitude, coherence phase, and gain between some reference latitude, chosen based on dynamical and statistical considerations, and all other latitudes between $11.75^{\circ} \mathrm{S}$ and $11.75^{\circ} \mathrm{N}$. These estimates and their confidence intervals are computed following Bendat and Piersol. To retain the meridional resolution needed to examine the meridional $\mathrm{SSH}$ structure of equatorial waves, spectral quantities from different latitudes are not averaged together. As a result, there are fewer degrees of freedom than in the power spectral estimates, and thus more frequencyband averaging ( 11 bands) is performed to improve the accuracy of the estimates. These calculations have only 22 degrees of freedom, but the reasonably high coherence between latitudinally separated locations due to the presence of equatorial modes leads to acceptable levels of uncertainty in the meridional profiles of gain and phase. For the coherence calculations, the wavenumber bandwidth is the same as for the power spectra $\left(1 / 124^{\circ}\right)$, and the frequency bandwidth is about $1 /(298.6$ days).

The meridional profiles of gain and phase for a given wavenumber-frequency band specify the meridional structure of SSH variability that is coherent with that at the reference latitude. The gain calculation yields an estimate of the meridional profile of the amplitude of variability in a given wavenumber-frequency band that is coherent with variability at the reference latitude. The coherence phase at each latitude relative to the reference latitude gives the meridional phase variation of the amplitude profile estimated from the gain calculation. To the extent that the variability is not statistically stationary in time and longitude, the results in each spectral band represent a composite wave, averaged over time and longitude.

\section{Theoretical background and terminology}

Interpretation and identification of equatorial modes in the observations is facilitated by having in mind some theoretical accounts of the dispersion relations and meridional structures of equatorial waves. Of particular interest for interpretation of the sea level signal of equatorial waves is the theoretical prediction for the pressure field. This section briefly reviews some relevant aspects of the theory of equatorial waves.

In the "classical theory" of equatorial waves, where the equations of motion for a shallow, homogeneous layer of fluid on an unbounded equatorial $\beta$ plane are linearized about a state of rest, the properties of equatorial waves take on a particularly simple form (e.g., Matsuno 1966; Moore and Philander 1977). The equations of motion can be combined into a single equation in meridional velocity, with eigenvalues that are positive, odd integers $(2 n+1=1,3,5, \ldots)$ and eigenfunctions that are Hermite functions. Wave motions, periodic in longitude and time, are organized into a set of discrete meridional modes. The $n=0$ eigenvalue corresponds to the mixed Rossby-gravity wave (or Yanai wave), and there are both Rossby and gravity wave roots for $n \geq 1$. The system also admits a Kelvin wave, sometimes called the $n=-1$ root (Matsuno 1966). The dispersion curves of the classical theory for first baroclinic-mode Rossby and Kelvin waves are shown in Fig. 1. An important point for what follows is that the pressure signal of modes with odd $n$ is symmetric about the equator; the pressure signal of modes with even $n$ is antisymmetric. This expectation from the classical theory will serve as a rough guide in interpreting waveinduced sea level variations, which result from pressure fluctuations associated with the wave modes.

Much work in the last few decades has focused on the deviation of the properties of equatorial waves in the real ocean from those of the classical theory, and most of this work has focused on the effects of the equatorial zonal current system on the dispersion and meridional structure of these waves (e.g., McPhaden and Knox 1979; Boyd, 1982a,b; McPhaden et al. 1986; Brossier 1987; McPhaden and Ripa 1990; Johnson 1993; Johnson and McPhaden 1993; Chelton et al. 2003; Lyman et al. 2005; Perez et al. 2005; and references therein). Examination of the effects of realistic equatorial currents usually involves linearization of the equations of motion about some more or less realistic representation of the meridional and vertical profile of the equatorial current system. In general, determining the dispersion curves and meridional eigenfunctions by this approach requires numerical solution of the resulting eigenvalue problem. For the first baroclinic-mode Kelvin wave, the equatorial currents are expected to have a modest effect on the phase speed, an appreciable effect on the meridional eigenfunctions for zonal and meridional ve- 


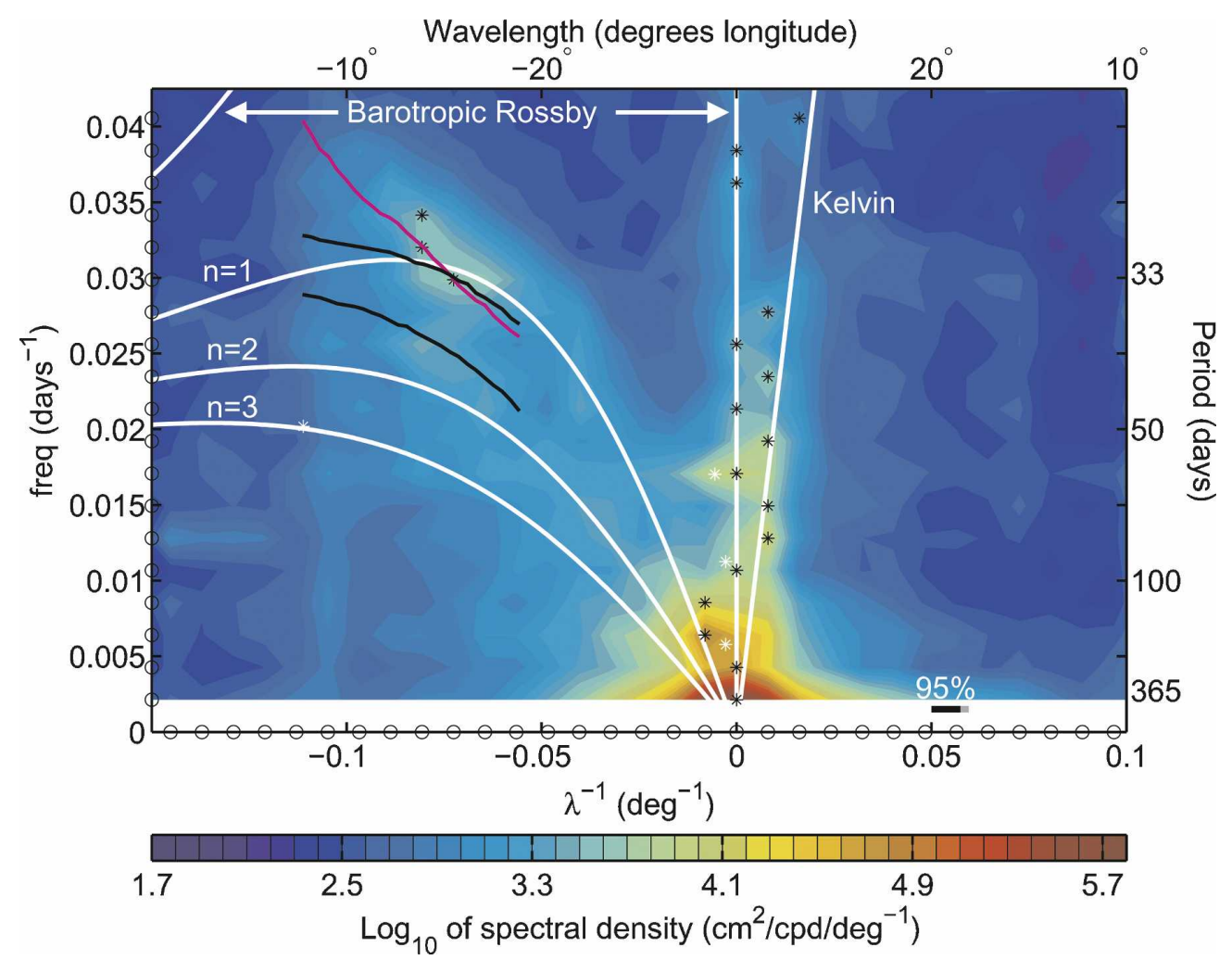

FIG. 1. $\log _{10}$ of power spectral density of SSH $\left(\mathrm{cm}^{2} \mathrm{cpd}^{-1} \mathrm{deg}^{-1}\right)$ averaged over $6.75^{\circ} \mathrm{S}-6.75^{\circ} \mathrm{N}$. Black asterisks mark spectral peaks within each frequency band; white asterisks mark expected tidal aliases in the TOPEX/Poseidon data (Schlax and Chelton 1994). The horizontal black line in the bottom right-hand side of each panel indicates the estimated $95 \%$ confidence interval (to be compared to the color scale), and the gray line indicates the additional uncertainty at $99 \%$ confidence. For reference, theoretical dispersion curves of the first baroclinic mode in a resting ocean are indicated by white lines, and a barotropic Rossby wave dispersion curve is also indicated. The black lines, from Lyman et al. (2005), show how the dispersion curves of the first- and second-meridional-mode Rossby waves are modified when the effects of mean flows are considered in a model of the first baroclinic mode. The pink line, also from Lyman et al., is a theoretical prediction for the real part of the dispersion relation of the tropical instability wave mode.

locity, and a relatively small effect on the meridional eigenfunction for pressure (McPhaden and Knox 1979).

For Rossby waves and other westward propagating variability, the zonal current system has a more dramatic effect on the phase speed and meridional eigenfunction for pressure (Chelton et al. 2003; Lyman et al. 2005; Perez et al. 2005). Chelton et al. (2003) and Perez et al. (2005) considered the effect of the mean flow on basin-scale, annual period, first meridional-mode Rossby waves and found that the westward zonal phase speed is reduced by $30 \%-50 \%$ and the sea surface height signal is larger north of the equator. Lyman et al. (2005) used the equatorial zonal currents of a realistic general circulation model (GCM) to examine the effect of the equatorial current system on tropical instability waves (TIWs) and other westward propagating modes of variability by projecting the linearized system of equations onto a set of baroclinic modes; this approach reduces the complexity of the vertical structure that is modeled but still allows interaction between the waves and the mean flow and is akin to similar analyses using 21/2-layer models (e.g., Donohue and Wimbush 1998). Lyman et al. (2005) offered estimates of the dispersion relation for westward propagating modes having wavelengths of $9^{\circ}-18^{\circ}$ longitude.

To give a rough idea of how the modal dispersion curves of the real ocean can differ from those of the classical theory, dispersion curves from Lyman et al. (2005, their Fig. 9) are also shown in Fig. 1. Two of the curves show how the dispersion properties of the first two Rossby modes are modified in the presence of a realistic mean flow when interaction of vertical modes is not allowed (i.e., when only one vertical mode is used in the projection), and one curve shows the real part of the dispersion relation for the unstable TIW mode, which can be interpreted as involving interaction of 
multiple vertical and meridional modes and the mean flow (Lyman et al. 2005).

Though the dispersion curves presented by Lyman et al. (2005) may be the most realistic to date, the dispersion relation obeyed by equatorial waves probably varies as a function of space and time. The dispersion curves from Lyman et al. in Fig. 1 were derived for a particular choice of a latitude-dependent and depthdependent zonal flow meant to be representative of the equatorial current system in the eastern Pacific just before the development of tropical instability waves. These curves are not expected to be fully representative of the dispersion curves in the Pacific Ocean, which should vary in response to spatial and temporal variations in currents, stratification, and possibly other factors such as forcing. For a limited band of wavenumbers and frequencies, Lyman et al. discuss how the dispersion properties of Rossby waves are affected by variations in the strength of a latitude-dependent and depthdependent profile of the zonal flow.

Some empirical generalizations can be distilled from the previous theoretical work on the meridional structure and dispersion relations of equatorial waves in the presence of the equatorial current system: 1) Though the meridional structure in pressure or sea level of equatorial modes is affected by the meridionally and vertically sheared zonal current system, these effects are not so profound as to make the meridional structures unrecognizably different from the modes of the classical theory. For example, a first meridional-mode Rossby wave in a realistic zonal flow (Chelton et al. 2003) has two maxima in sea level amplitude that are located at latitudes roughly opposite about the equator and are in phase, as expected from the classical theory. However, unlike the classical theory, the two maxima do not have the same amplitude. 2) Though the mean flow can have a substantial effect on the dispersion relations of equatorial waves, and on those of Rossby waves in particular, the dispersion curves are nonetheless expected to resemble those of the classical theory (McPhaden and Knox 1979; Brossier 1987; Chelton et al. 2003; Lyman et al. 2005).

Based on these expectations, the dispersion curves of the classical shallow-water theory will be used here as a guide to aid in interpretation of the observed spectral characteristics of equatorial sea level variations. Details of these dispersion curves for a linear, resting ocean are not relied on for any quantitative inference, and their use is not meant to suggest that the shallow-water theory gives an adequate description of the dynamics. The dispersion curves are meant to provide a point of reference for orientation in the wavenumber-frequency domain. The appropriate value of the gravity wave speed is uncertain owing to spatial and temporal variations in the stratification; over most of the equatorial Pacific, the time-mean gravity wave speed of the first baroclinic mode falls between 2.4 and $3.0 \mathrm{~m} \mathrm{~s}^{-1}$ (Chelton et al. 1998). The value used to generate the curves in Fig. 1 and later figures is $2.7 \mathrm{~m} \mathrm{~s}^{-1}$.

\section{Results}

Values of wavenumber and frequency for which the SSH power spectrum for the $6.75^{\circ} \mathrm{S}-6.75^{\circ} \mathrm{N}$ equatorial belt (Figs. 1,2) is significantly above the background are expected to indicate the variance-weighted mean location of dispersion curves for equatorial waves (and also other types of SSH variations that occur preferentially at particular wavenumber-frequency values, such as the direct response to atmospheric forcing). As might be anticipated, the highest power levels are found at scales on the order of the basin scale and at low frequencies (Fig. 1). Since the spectral resolution is too coarse to distinguish between the various modes at these small wavenumbers and frequencies, where all of the theoretical dispersion curves converge toward zero wavenumber and zero frequency, the low-wavenumber, low-frequency variability will not be considered here, and power contour levels in later figures will not be shown for this variability.

Although the Pacific basin is too narrow to allow the Kelvin wave to be well resolved in wavenumber, the dispersion curve for the baroclinic Kelvin wave is nonetheless apparent in the SSH power spectrum for the equatorial belt (Fig. 2): there are significant spectral peaks at low, positive wavenumbers corresponding to eastward propagation. These elevated power levels lie close to the Kelvin wave dispersion curve. Theoretical considerations of the effect of the mean zonal currents on propagation of Kelvin waves in the period band considered here (McPhaden and Knox 1979) suggest that the Kelvin wave should be slightly Doppler shifted, but this relatively small shift cannot be resolved. Because of inherent limitations on wavenumber resolution, any Kelvin wave phase speed between 2.2 and $4.5 \mathrm{~m} \mathrm{~s}^{-1}$ would be consistent with most of the observed spectral peaks, so this analysis does not give any particularly useful insight into the phase speed of the Kelvin wave variability. Despite this, it is reasonable to conclude that spectral peaks associated with long-wavelength variability propagating eastward at about $3 \mathrm{~m} \mathrm{~s}^{-1}$ are due to Kelvin waves. (Additional evidence is given in section 5.)

Aside from the high power levels associated with Kelvin waves and basin-scale variability, the highest power at periods less than about 100 days occurs in an 


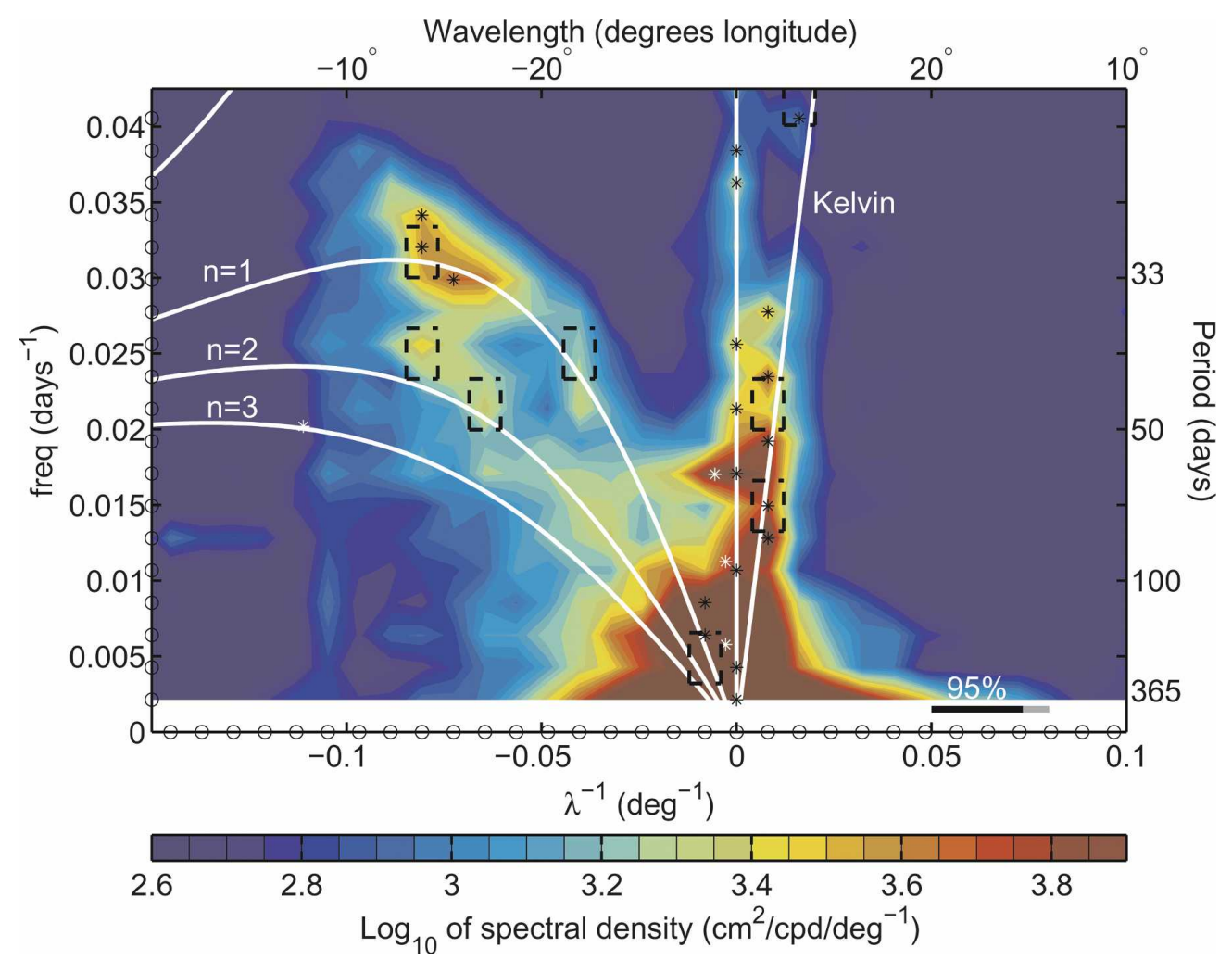

FIG. 2. $\log _{10}$ of power spectral density of SSH $\left(\mathrm{cm}^{2} \mathrm{cpd}^{-1} \mathrm{deg}^{-1}\right.$ ) averaged over $6.75^{\circ} \mathrm{S}-6.75^{\circ} \mathrm{N}$ (as in Fig. 1 , except with a smaller range of contoured values). Theoretical (zero mean flow) dispersion curves for the first baroclinic mode (white lines) are the same as in Fig. 1. The boxes made with dashed lines indicate wavenumber-frequency bands for which the meridional structure of SSH variability is examined in section 5. The horizontal black line in the bottom right-hand side of each panel indicates the estimated $95 \%$ confidence interval (to be compared to the color scale), and the gray line indicates the additional uncertainty at $99 \%$ confidence.

isolated spectral peak associated with tropical instability waves (TIW) at periods of 29.2-33.4 days and wavelengths of $12.4^{\circ}-15.5^{\circ}$ longitude (Fig. 2). Power levels are above the background over a somewhat broader region. Although the spectral peak occurs over a narrower range of wavenumbers and frequencies and at slightly larger periods and wavelengths than TIW variability observed in some previous analyses (e.g., Qiao and Weisberg 1995, and references therein), it seems clear that this spectral peak is a manifestation of the energetic tropical instability waves that dominate the monthly time scale variability of the equatorial Pacific Ocean.

At negative (i.e., westward) wavenumbers, there is generally elevated power near the classical Rossby wave dispersion curves (Fig. 2). Remarkably, though, spectral peaks are present near both $n=1$ and $n=2$ Rossby wave dispersion curves with a statistically significant trough between them. That is, there is evidence of at least two discrete Rossby wave modes. [While there is overwhelming observational evidence of the existence of the $n=1$ mode, the author is not aware of direct and unambiguous observational evidence of a second meridional-mode Rossby wave in the ocean or atmosphere. However, there is evidence suggesting the presence of a second meridional-mode Rossby wave in the ocean (e.g., Delcroix et al. 1994; Boulanger and Menkes 1995, 1999; Polito and Sato 2003) and atmosphere (e.g., Roundy and Frank 2004b)]. Because the first baroclinic-mode $n=2$ Rossby wave dispersion curve is expected to lie near the second baroclinicmode $n=1$ Rossby wave curve (e.g., Zang et al. 2002), it is not possible to determine which of these two waves contributes more to the power near the theoretical $n=$ 2 curve by inspection of this power spectrum. However, it is worth noting that a second baroclinic-mode wave must be about 3 times more energetic (in terms of internal vertical velocity) than a first baroclinic-mode wave so as to make an equal contribution to sea level amplitude (Wunsch and Gill 1976). Later in this section and in section $5 \mathrm{c}$, further insight into the nature of this second mode will be gained by examining the meridional structure and symmetry properties of this mode. 


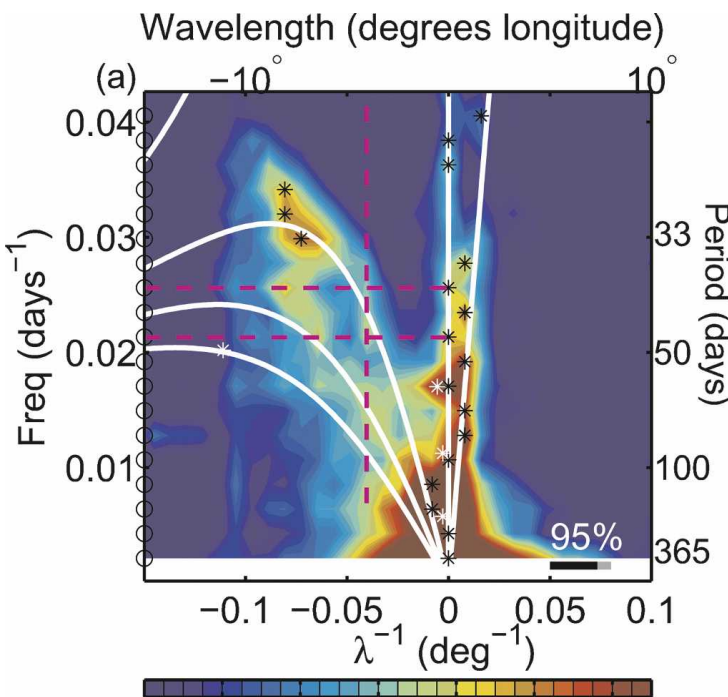

$\begin{array}{lllllll}2.6 & 2.8 & 3 & 3.2 & 3.4 & 3.6 & 3.8\end{array}$
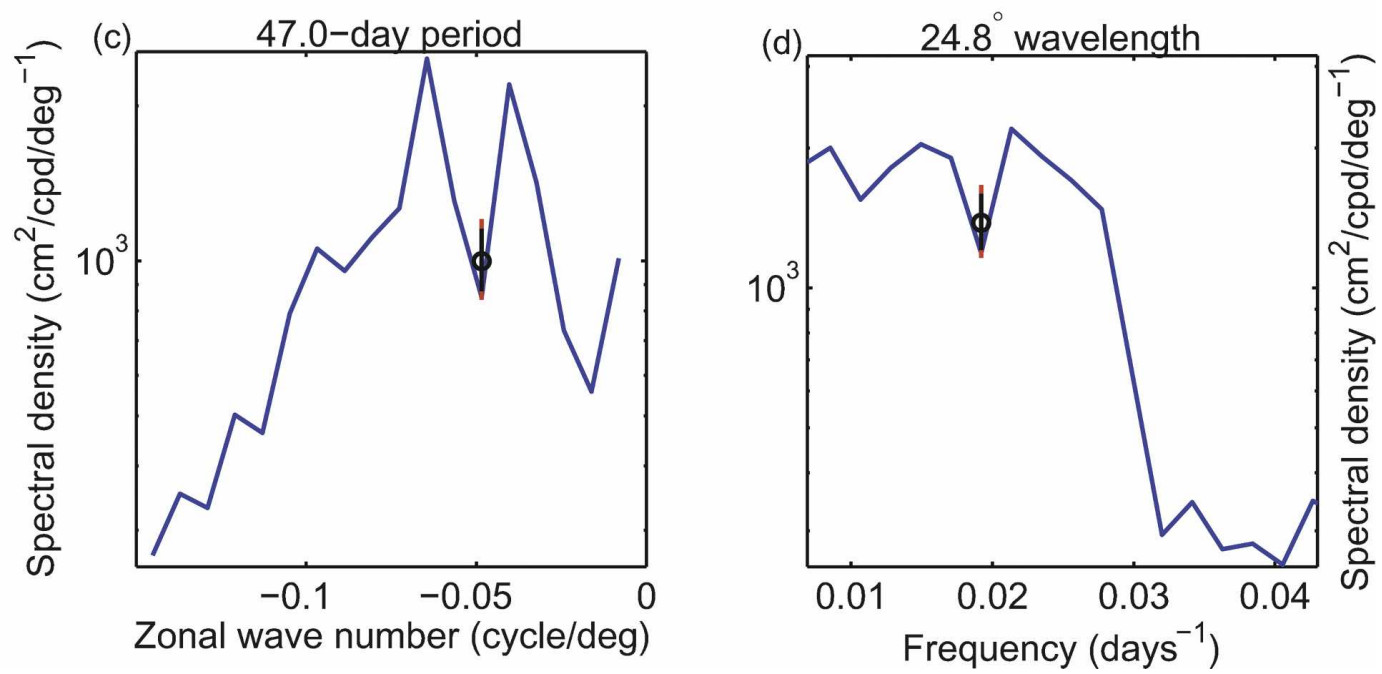

FIG. 3. (a) $\log _{10}$ of power spectral density of SSH averaged over $6.75^{\circ} \mathrm{S}-6.75^{\circ} \mathrm{N}$ (as in Fig. 2). The pink lines indicate wavenumber and frequency values for spectra shown in the other three panels. Power spectral density of SSH for westward propagating variability in the (b) 39.1-day period band [top horizontal pink line in (a)], (c) 47-day period band [bottom horizontal pink line in (a)], and (d) $24.8^{\circ}$ wavelength band [vertical pink line in (a)]. Two error bars are shown in (b), (c), and (d): the black line is the estimated $95 \%$ confidence interval, and the red line is the $99 \%$ confidence interval. In (b), (c), and (d), the error bars have been placed in the trough between the $n=1$ and $n=2$ Rossby wave peaks. The trough between the Rossby wave peaks near the $n=1$ and $n=2$ dispersion curves is statistically significant at $99 \%$ confidence along each of these sections.

The spectral gap or trough between the spectral peaks lying near the classical $n=1$ and $n=2$ Rossby wave dispersion curves is statistically significant (Fig. $3)$. Whether viewed as a function of wavenumber within the 39.1-day period band (Fig. 3b) or the 47-day period band (Fig. 3c) or as a function of frequency in the $24.8^{\circ}$ wavelength band (Fig. $3 \mathrm{~d}$ ), the trough between the $n=1$ and $n=2$ Rossby wave peaks is significant at $99 \%$ confidence (red error bars in Figs. 3bd). Even if the number of degrees of freedom were, pessimistically, assumed to be half of the estimated 392 degrees of freedom, the trough between the $n=1$ and $n=2$ Rossby wave dispersion curves would still be significant at $99 \%$ confidence (not shown). Although it is not yet clear to what extent the power near the $n=$ 2 Rossby wave curve is associated with variability resembling the $n=2$ theoretical mode, the $6.75^{\circ} \mathrm{S}-6.75^{\circ} \mathrm{N}$ power spectrum of SSH gives strong evidence for the existence of at least two distinct equatorial Rossby wave modes. 
The relative contributions to the average SSH variance over the equatorial belt (Fig. 2) from variability in the Northern and Southern Hemispheres can be quantified by comparing power spectra computed over $0.25^{\circ}-6.75^{\circ} \mathrm{N}$ and $0.25^{\circ}-6.75^{\circ} \mathrm{S}$ (Fig. 4). While the Kelvin wave is associated with roughly equal SSH variance north and south of the equator, the Rossby wave and TIW bands clearly have more power north of the equator, consistent with earlier studies (e.g., Kessler and McCreary 1993; Chelton et al. 2003; Perez et al. 2005; Lyman et al. 2005). Assuming that the power near the Rossby wave curves is associated with equatorial Rossby waves, this difference in SSH power between the Northern and Southern Hemispheres is a clear indication that the meridional amplitude profiles of the Rossby waves are asymmetric, because variability that is purely symmetric or antisymmetric about the equator would necessarily have equal power north and south of the equator. There are distinct ridges and a trough near the $n=1$ and $n=2$ Rossby wave dispersion curves in both hemispheres, and the trough has less power than the ridges at $95 \%$ confidence or better in both hemispheres along the same three sections of wavenumberfrequency space considered in Fig. 3 for the $6.75^{\circ} \mathrm{S}-$ $6.75^{\circ} \mathrm{N}$ spectrum of unfiltered SSH variability.

Even though Fig. 4 and previous work (e.g., Lukas and Firing 1985; Kessler and McCreary 1993; Chelton et al. 2003; Perez et al. 2005; Lyman et al. 2005) indicate that the SSH signal of equatorially trapped Rossby waves in the Pacific is asymmetric, the symmetric/ antisymmetric filtering procedure described in section 2 can still successfully isolate modes that are symmetric or antisymmetric in the classical theory, so long as the actual modes of variability are roughly symmetric or antisymmetric. Annual-period, $n=1$ Rossby waves, though asymmetric, are roughly symmetric in the sense that the peak amplitude in SSH south of the equator is of the same order of magnitude as that north of the equator, being only about two times smaller (Chelton et al. 2003; Perez et al. 2005). If the amplitude of a signal in the Northern Hemisphere is twice that found at the corresponding latitude in the Southern Hemisphere and the signals have the same phase, then the symmetric filtered signal will have nine times more power than the antisymmetric signal [i.e., $(2+1)^{2} /(2-$ $\left.1)^{2}=9\right]$; similarly, if the signals are $180^{\circ}$ out of phase, the antisymmetric power will be nine times larger than the symmetric power. Even if the amplitude north of the equator is five times greater than that south of the equator, the symmetric and antisymmetric spectra will differ by more than a factor of 2 . Thus, the symmetry filtering procedure is expected to be useful in isolating waves resembling $n=2$ Rossby waves from those re- sembling $n=1$ Rossby waves, despite asymmetries in their meridional structure, so long as the phase relation between variability on opposite sides of the equator resembles expectations based on the classical theory. (If variability on opposite sides of the equator is neither in phase nor out of phase, then the difference between symmetric and antisymmetric signals will be less for a given ratio of peak amplitudes.)

The power spectra of the symmetric and antisymmetric fields (Fig. 5) suggest that much of the power near the $n=2$ Rossby wave dispersion curve is associated with SSH variability that is roughly antisymmetric about the equator, as expected for the $n=2$ mode. Power near the $n=2$ curve is attenuated in the symmetric spectrum, while in the antisymmetric spectrum each period band between 40 and 70 days has its maximum power near the $n=2$ Rossby wave dispersion curve. The ridge of power present near the $n=1$ Rossby wave dispersion curve in the spectra of unfiltered data (Figs. 2-4) is more clearly seen in the symmetric spectrum and it is not present in the antisymmetric spectrum. (The relative power in the symmetric and antisymmetric fields is examined more quantitatively below.)

In the TIW band, there is significant power in both the symmetric and antisymmetric fields, but there is more power in the symmetric field. This result could be anticipated from the analytical model of Lyman et al. (2005) or many other previous studies since the TIWinduced SSH perturbation is of the same sign on both sides of the equator but is of much larger amplitude in the Northern Hemisphere. (That is, the TIW SSH signal is meridionally asymmetric, but it is more nearly symmetric than antisymmetric.)

At large zonal wavelengths (small wavenumbers), there is much more power in the symmetric spectrum than in the antisymmetric spectrum. That the Kelvin wave cannot be detected in the antisymmetric field suggests that Kelvin waves are very nearly symmetric about the equator. This is consistent with the classical theory and also with expectations based on the shearmodified theory (McPhaden and Knox 1979), which suggests that the effect of meridionally sheared zonal currents on the pressure eigenfunction of the Kelvin wave should be small in the period band considered here. Some of the low-wavenumber power in the symmetric spectrum could also result from direct forcing of sea level by symmetric atmospheric Kelvin waves, Rossby waves, and the Madden-Julian oscillation. The zonal wavenumber of these forms of atmospheric variability cannot be distinguished from zero in this analysis because these atmospheric motions generally have a 

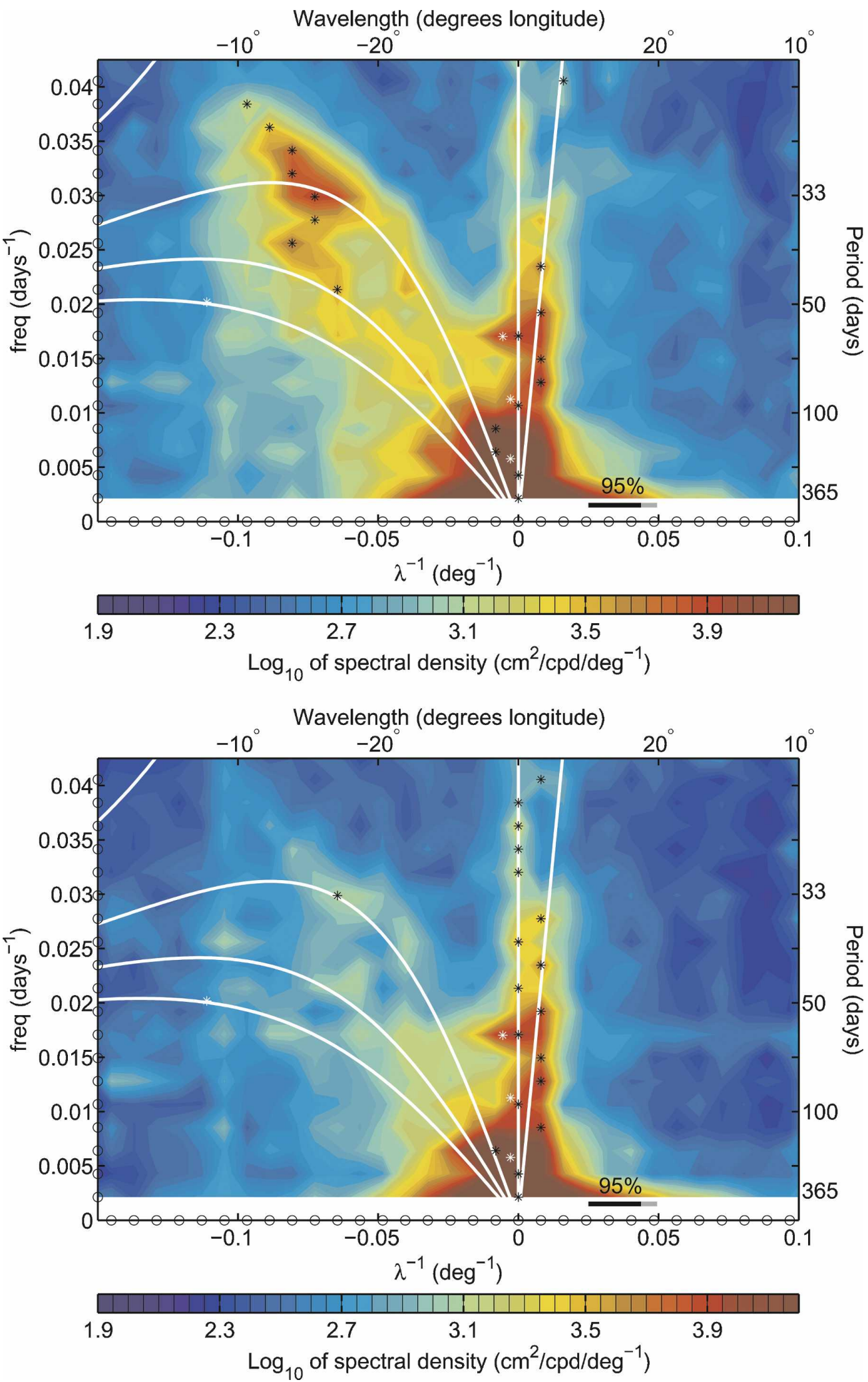

FIG. 4. $\log _{10}$ of power spectral density $\left(\mathrm{cm}^{2} \mathrm{cpd}^{-1} \mathrm{deg}^{-1}\right)$ of $\mathrm{SSH}$, averaged over (top) $0^{\circ}-6.75^{\circ} \mathrm{N}$ ("Northern Hemisphere"), and (bottom) $0^{\circ}-6.75^{\circ}$ S ("Southern Hemisphere"). Westward-propagating SSH variations with periods less than 100 days have higher variance in the Northern Hemisphere, which suggests that these variations are asymmetric about the equator. See the caption of Fig. 1 for description of confidence intervals, lines, and symbols. 

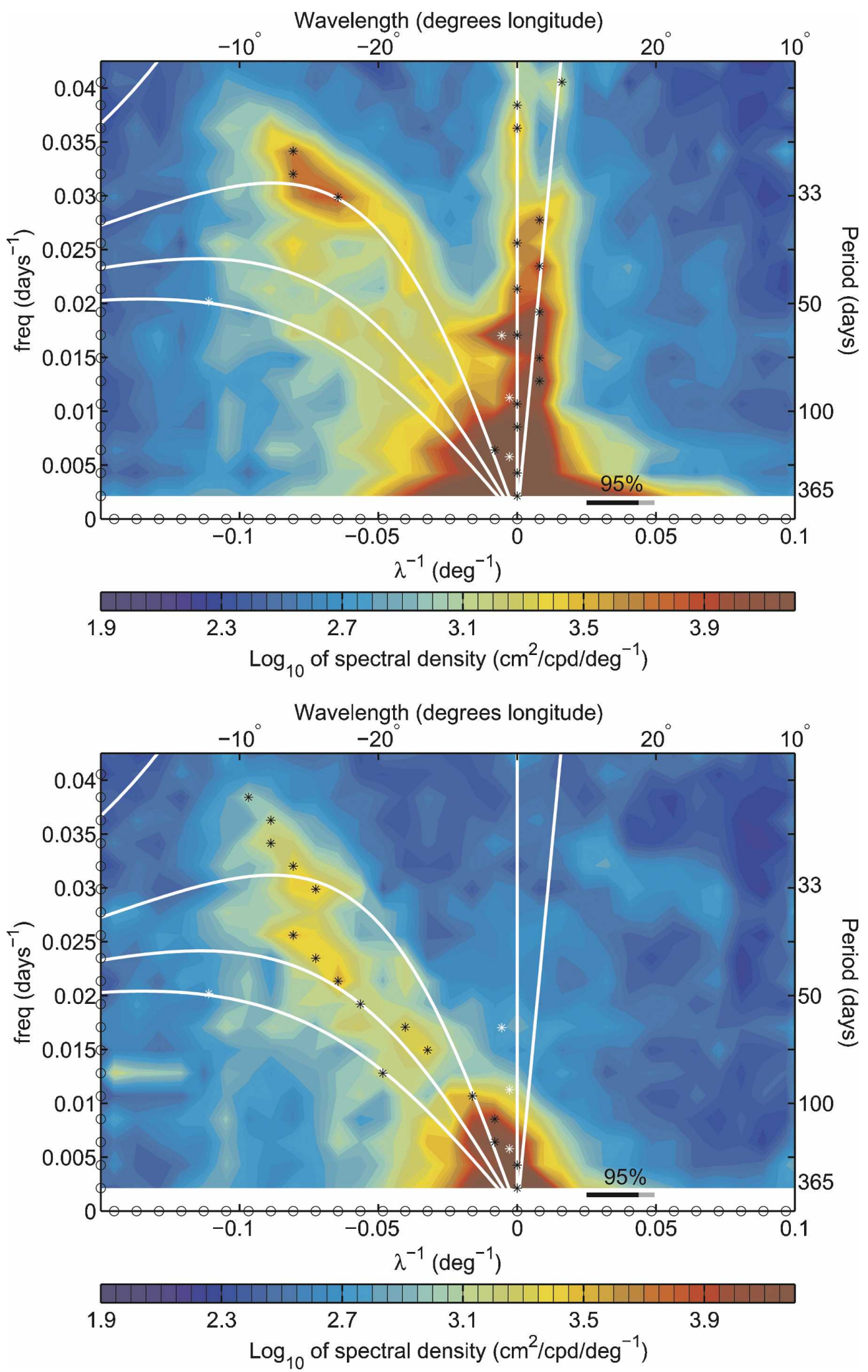

FIG. 5. $\log _{10}$ of power spectral density $\left(\mathrm{cm}^{2} \mathrm{cpd}^{-1} \mathrm{deg}^{-1}\right)$ of (top) symmetric SSH variability, averaged over $6.75^{\circ} \mathrm{S}-6.75^{\circ} \mathrm{N}$, and (bottom) antisymmetric SSH variability, averaged over $6.75^{\circ} \mathrm{S}-6.75^{\circ} \mathrm{N}$. The symmetric spectrum shows elevated power near the dispersion curves of the theoretically symmetric Kelvin wave and $n=1$ Rossby wave, while the antisymmetric spectrum shows elevated power near the dispersion curve of the theoretically antisymmetric $n=2$ Rossby wave at periods of 40-70 days. (See the caption of Fig. 1 for description of confidence intervals, lines, and symbols.) 


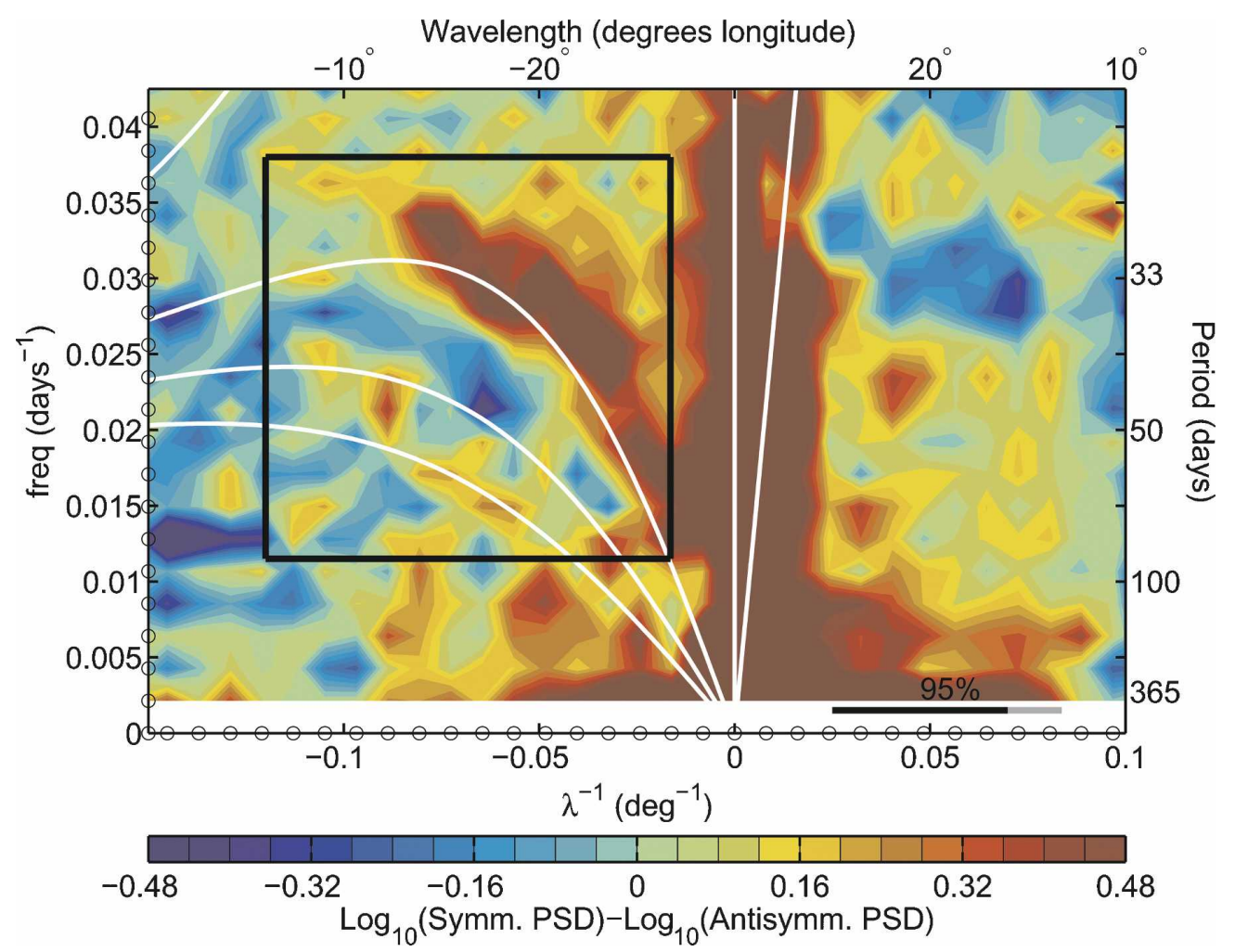

FIG. 6. $\log _{10}$ of the ratio of symmetric power to antisymmetric power, equivalent to $\log _{10}($ symmetric power $)-\log _{10}$ (antisymmetric power). The contours shown range over values of symmetric power about 3 times less than antisymmetric power (i.e., $10^{-0.48} \approx 1 / 3$ ) to values of symmetric power about 3 times greater than antisymmetric power (i.e., $10^{0.48} \approx 3$ ). The estimated $95 \%$ confidence interval is indicated by a horizontal black line, and the gray line indicates the additional uncertainty at $99 \%$ confidence. Values greater than about 0.17 have more power in the symmetric spectrum than in the antisymmetric spectrum at $95 \%$ confidence or better, and vice versa for values less than -0.17 . In the region of the spectral domain associated with equatorial Rossby waves that are well resolved (indicated by a black rectangle), there is some suggestion of wave modes that are alternately dominantly symmetric or dominantly antisymmetric.

zonal scale as large as or larger than the Pacific. [See Wheeler and Kiladis (1999) or Roundy and Frank (2004a) for a description of the wavenumber-frequency spectra and symmetry properties of these forms of atmospheric variability.] Barotropic Rossby and Kelvin waves in the ocean might also contribute to variability in the zero-wavenumber band.

More quantitative information about the relative magnitude of symmetric and antisymmetric variations in SSH can be extracted by considering the ratio of symmetric power to antisymmetric power. The logarithm of this ratio is identical to the difference of the logarithms of the symmetric and antisymmetric power, a fact that allows a graphical presentation in which statistically significant differences between symmetric and antisymmetric SSH power can be easily assessed (Fig. 6). As is also clear from inspection of Fig. 5, the symmetric power far exceeds the antisymmetric power near the $n=1$ Rossby wave curve and the difference be- tween the two is statistically significant at $95 \%$ confidence. Near the $n=2$ theoretical Rossby wave curve (but at slightly higher frequencies), the antisymmetric power exceeds the symmetric power, and this difference is statistically significant at most of these points. The $n=2$ dispersion curve in the Pacific is expected to lie at slightly higher frequencies than the corresponding dispersion curve of the zero-mean flow theory because of the effect of the equatorial current system [e.g., see the shear-modified dispersion curve reproduced from Lyman et al. (2005) in Fig. 1].

Interestingly, there is some suggestion of another region of wavenumber-frequency space at slightly lower frequencies, near the $n=3$ curve, that exhibits dominantly symmetric power, and the symmetry preference reverses again at even lower frequencies. This may be evidence of higher meridional modes, but it becomes increasingly difficult to interpret this variability in sea level moving to lower frequencies where several verti- 
cal and meridional modes are expected to exist. No further attention will be devoted to this variability here.

\section{Discussion: Meridional structure of the observed wave modes}

The wavenumber-frequency spectra presented in Figs. 2-6 indicate the presence of at least two equatorially trapped Rossby wave modes. A mode of variability resembling the $n=1$ theoretical Rossby wave mode has been previously observed in several datasets and contexts, and it is clear that this mode, which is quasi symmetric in sea level, is apparent in the spectra of SSH presented here. A mode of variability resembling the $n=2$ Rossby wave mode has not been previously isolated in the ocean or atmosphere (as far as the author knows), and interpretation of the spectra is made difficult by the fact that the first baroclinic-mode $n=2$ dispersion curve is expected to lie near the second baroclinic-mode $n=1$ dispersion curve. On one hand, the antisymmetric SSH spectrum has a statistically significant ridge of power that lies near the first baroclinicmode $n=2$ dispersion curve, which suggests that a mode resembling the $n=2$ mode has been successfully isolated. On the other hand, the symmetric spectrum also has elevated power at some locations near the $n=$ 2 dispersion curve, and at some wavenumbers near the $n=2$ dispersion curve, the symmetric power is as large as the antisymmetric power. Thus, special care must be taken in trying to determine what this second mode of variability is.

One way of gaining further insight into the wave modes detected in the power spectra (Figs. 2-6) is to examine the meridional structure of the variability (i.e., the amplitude and phase as a function of latitude) in the wavenumber-frequency bands corresponding to each mode. This can be accomplished by computing the coherence amplitude, coherence phase, and gain of SSH at each latitude against the SSH signal at some reference latitude as described in section 2. This approach extracts variability in a wavenumber-frequency band coherent with that at the reference latitude. The particular wavenumber-frequency bands for which the meridional structure is examined are indicated in Fig. 2.

Before examining the meridional structure of variability that could be associated with the $n=2$ mode, it will be instructive to examine the structure of the Kelvin waves and $n=1$ Rossby waves. By comparing the estimated meridional structure of these relatively more well-studied waves to theory and previous observations, the method will be shown to produce sensible results, which will increase confidence in the results obtained for the second Rossby wave mode.

\section{a. The Kelvin wave}

For examination of the meridional structure of Kelvin waves, the coherence amplitude, gain, and phase for each latitude on $11.75^{\circ} \mathrm{S}-11.75^{\circ} \mathrm{N}$ were computed against $0.25^{\circ} \mathrm{N}$ because the Kelvin wave signal in SSH is expected to be largest near the equator. In section 4, energetic variability near the Kelvin wave dispersion curve was resolved away from zero wavenumber at periods less than about 70 days. Here, the meridional sea level structure of Kelvin wave variability in the 67-, 46-, and 24-day period bands is examined.

Intraseasonal (30-90 days) Kelvin waves in the Pacific have received considerable attention (Enfield 1987; McPhaden and Taft 1988; Johnson 1993; Johnson and McPhaden 1993; Kessler et al. 1995; Kessler and McPhaden 1995; Hendon et al. 1998; Kutsuwada and McPhaden 2002; Zang et al. 2002; Cravatte et al. 2003; Roundy and Kiladis 2006) and thus provide a useful point of reference for examination of the meridional sea level structure. These waves are forced in the western and central Pacific by intraseasonal variations in the zonal wind stress that are associated with the MaddenJulian oscillation (MJO), but the dominant period of the Kelvin waves, as measured by thermocline depth spectra, is about 70 days, whereas the dominant period for zonal wind stress variations associated with the MJO is 40-50 days (Kessler et al. 1995; Hendon et al. 1998). The SSH spectra here (e.g., Fig. 2) are consistent with the previous studies showing more power at 50-90 day periods than at $30-50$ day periods. There are few, if any, detailed accounts of the meridional structure of these prominent intraseasonal Kelvin waves, but Kutsuwada and McPhaden (2002) and Cravatte et al. (2003) have tangentially addressed the meridional structure in attempting to determine the wave speed by fitting observed sea level variability at these periods to the wave-speed-dependent Gaussian profile of the classical theory.

In the wavenumber-frequency band corresponding to basin-scale ( $124^{\circ}$ wavelength band), 60-75-dayperiod Kelvin waves, SSH variability at the "equator" $\left(0.25^{\circ} \mathrm{N}\right)$ is coherent at $95 \%$ confidence with that at all latitudes between $4.75^{\circ} \mathrm{S}$ and $6.25^{\circ} \mathrm{N}$, and there are a few scattered locations as far away as $11.75^{\circ} \mathrm{S}$ where significant coherence was also detected. (Gain and phase estimates in Fig. 7 and later figures are shown only at latitudes where the coherence amplitude of SSH against that at the reference latitude is significant at the $95 \%$ confidence level or better, i.e., where the coherence amplitude exceeds 0.51.) The meridional amplitude structure (Fig. 7a) closely resembles the Gaussian profile predicted by the classical theory (e.g., Matsuno 

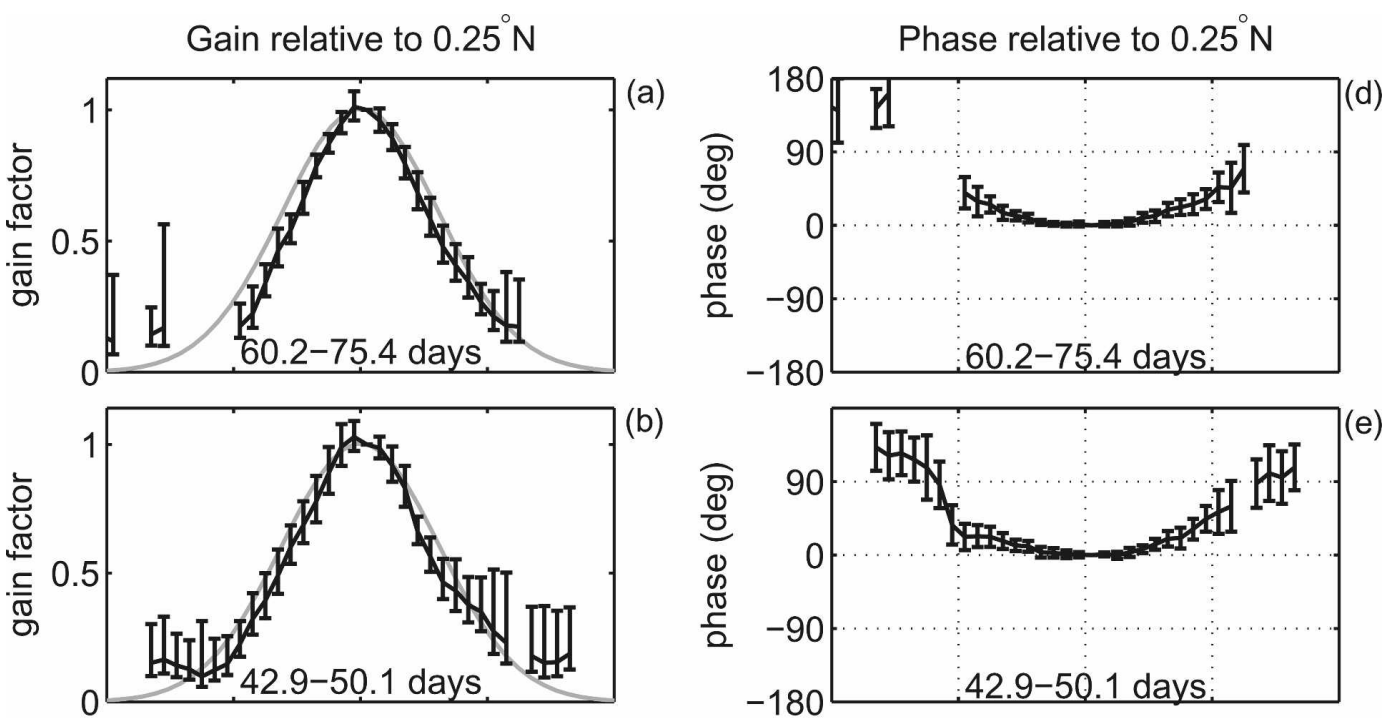

(b)
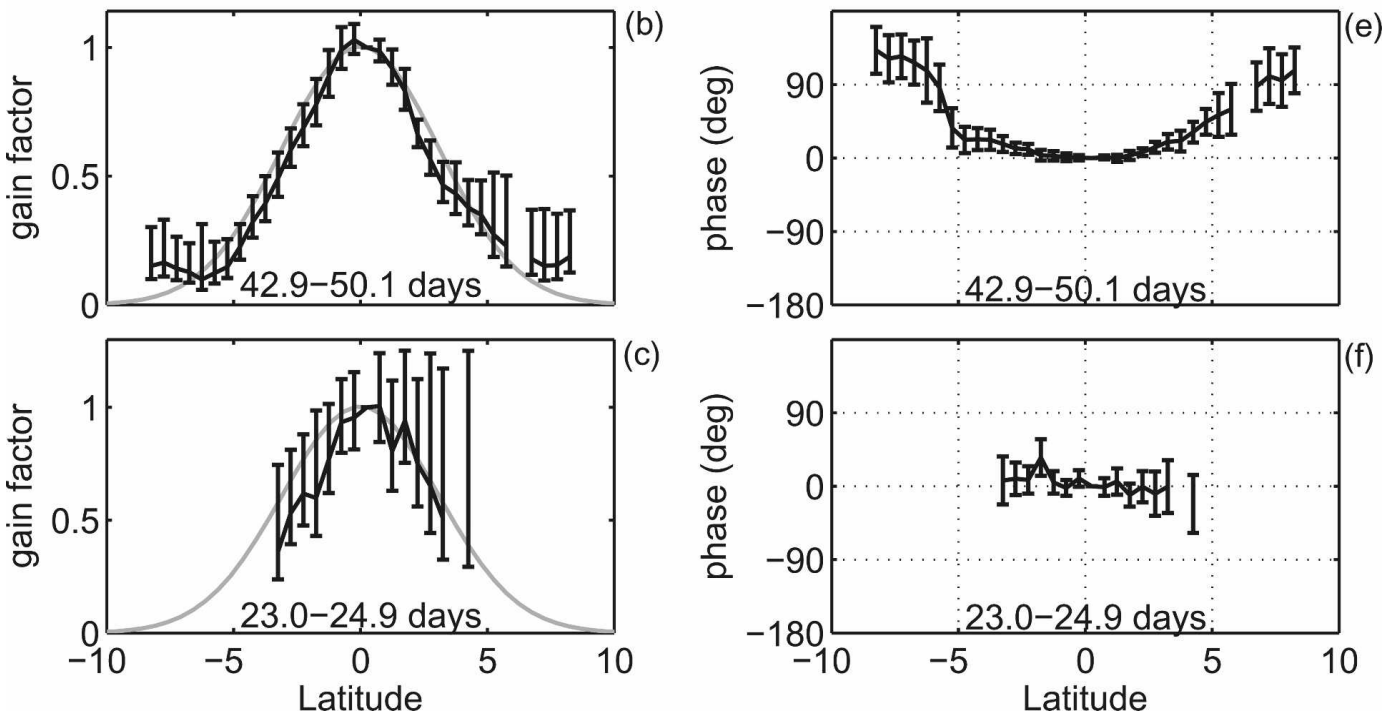

(c)

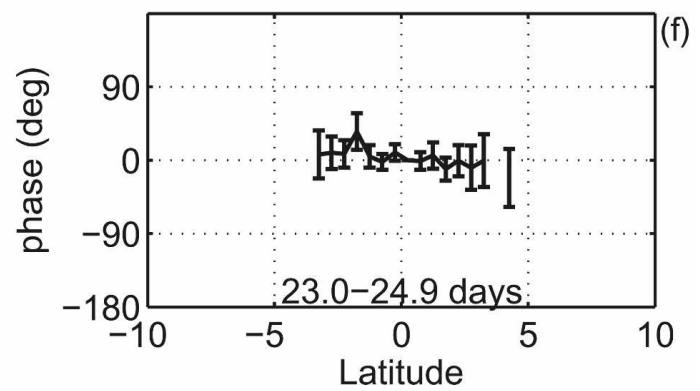

FIG. 7. Meridional profiles of SSH (a)-(c) amplitude and (d)-(f) phase relative to $0.25^{\circ} \mathrm{N}$ for points on the Kelvin wave dispersion curve in Fig. 2. Positive phase angles lead. The period band is indicated in each panel. Values are shown only at latitudes where the SSH variability was coherent with that at the reference latitude at $95 \%$ confidence or better, and the error bars are estimated 95\% confidence intervals. In (a), (b), and (c), the meridional profile of amplitude expected from the classical theory is shown as a gray line. Theoretical profiles were calculated using a Kelvin wave speed of $2.7 \mathrm{~m} \mathrm{~s}^{-1}$; smaller values would reduce the $e$-folding scale.

1966). ${ }^{1}$ Within a few degrees of the equator, the variability is essentially in phase but, moving away from the equator, the coherent SSH variability increasingly leads that at the equator (Fig. 7d).

The meridional profiles of amplitude and phase are similar in the 43-50-day period band (Figs. $7 \mathrm{~b}$ and 7e). The meridional amplitude profile is not significantly different from that for the 60-75-day period band, but

\footnotetext{
${ }^{1}$ The theoretical Kelvin wave profile is dependent on the gravity wave speed, which is $2.0-3.0 \mathrm{~m} \mathrm{~s}^{-1}$ for the first baroclinic mode (e.g., Chelton et al. 1998). The wave speed used to calculate the theoretical curves in Fig. 7 was chosen to be $2.7 \mathrm{~m} \mathrm{~s}^{-1}$, but a better fit to the observed profile can be obtained with a smaller speed. The Kelvin wave profile could also be affected by other factors such as mean flow, forcing, and contributions from higher baroclinic modes, so tuning the choice of wave speed to produce a better fit seems unjustified. The meridional profile inferred from the observations does not depend on assumptions about any of these factors.
}

there are more latitudes (i.e., all but one on $8.25^{\circ} \mathrm{S}-$ $8.25^{\circ} \mathrm{N}$ ) where the $43-50$-day period band variability is coherent with that at the equator. Between about $6.25^{\circ} \mathrm{S}-6.25^{\circ} \mathrm{N}$, where the Kelvin wave mode of classical theory is more than $10 \%$ of its amplitude at the equator, the observed meridional profile of the amplitude of the Kelvin wave in this wavenumber-frequency band is consistent with the theoretical prediction from a linearization about zero mean flow (e.g., Matsuno 1966). The more realistic theoretical study of McPhaden and Knox (1979) suggests that the equatorial current system should have little effect on the meridional structure of the pressure signal of the Kelvin wave in the period bands considered in this study, and Fig. 7 supports that conclusion.

The 46-day Kelvin wave's SSH signal is in phase over $2^{\circ} \mathrm{S}-2^{\circ} \mathrm{N}$, and the phase leads that at the equator by a progressively larger amount with distance from the equator, reaching a phase angle of about $115^{\circ}$ at $8^{\circ} \mathrm{S}$ 
and $8^{\circ} \mathrm{N}$. It is not obvious that the off-equatorial phase angles (or the gain outside of about $4^{\circ} \mathrm{S}-4^{\circ} \mathrm{N}$ ) should be interpreted as a dynamical characteristic of the Kelvin wave because the coherent variability detected off of the equator may be forced directly by the same largescale winds that force the basin-scale, intraseasonal Kelvin waves (Kessler et al. 1995; Hendon et al. 1998). Large-scale wind forcing coherent with the Kelvin wave variability might explain the fact that the coherent amplitude variability is about $15 \%$ of that at the equator as far away as $10.75^{\circ} \mathrm{S}$, which is inconsistent with any theory of free equatorial Kelvin waves. By solving the linearized shallow-water equations with forcing on an unbounded $\beta$ plane, it can be shown that an amplitude and phase profile similar to the observed one can be produced if the wind stress forcing at this wavenumber and frequency has symmetric zonal and antisymmetric meridional components (not shown) meant to mimic the observed surface wind signal of the MJO (Hendon and Salby 1994). Thus, it is at least plausible that the statistically robust, coherent signal in SSH detected away from the equator is due to the forced nature of the intraseasonal Kelvin waves.

While intraseasonal Kelvin waves with basin-scale wavelengths have been observed by many investigators, there have been fewer observations of Kelvin waves with periods of a few weeks (but see Eriksen 1982). For this reason, the meridional structure in the 24-day period and $62^{\circ}$ wavelength band is also shown in Fig. 7. In this wavenumber-frequency band there is an isolated and statistically significant spectral peak in the spectrum for the $6.75^{\circ} \mathrm{S}-6.75^{\circ} \mathrm{N}$ equatorial belt (Fig. 2), and variability in $\mathrm{SSH}$ is coherent with that at $0.25^{\circ} \mathrm{N}$ over $3.25^{\circ} \mathrm{S}-4.25^{\circ} \mathrm{N}$ at $95 \%$ confidence or better. The meridional SSH structure of the 24-day Kelvin wave is consistent with the Gaussian structure predicted by classical theory and with the meridional structure of the lower-frequency intraseasonal Kelvin waves, but the $95 \%$ confidence intervals are large (Fig. $7 \mathrm{c}$ ). The wave is essentially in phase over the latitude band where coherent SSH variability was detected (Fig. 7d), although the phase at $1.75^{\circ} \mathrm{S}$ is different from zero at more than $95 \%$ confidence.

\section{b. The $n=1$ Rossby waves and tropical instability waves}

The power spectra indicated the presence of a welldefined ridge of power lying near the $n=1$ dispersion curve (Figs. 2-6), and the dominance of symmetric power over antisymmetric power near this curve indicates that this variability is more nearly symmetric than antisymmetric about the equator. The meridional structure of SSH variability associated with this spectral peak was examined by computing the gain and coherence phase against the SSH variability at $5.25^{\circ} \mathrm{N}$. (The results did not change qualitatively when the coherence calculations were carried out against latitudes between $6^{\circ} \mathrm{S}$ and $6^{\circ} \mathrm{N}$.) Results are reported here for the three spectral bands indicated by boxes near the $n=1$ dispersion curve in Fig. 2.

In the $22.5^{\circ}-27.6^{\circ}$-wavelength, 37.5-42.9-day period band, where the symmetric and antisymmetric spectra suggested that the variability is nearly symmetric about the equator, the meridional structure is, indeed, more symmetric than antisymmetric (Figs. 8a and 8d). The amplitude peaks of this mode of variability are found near $5^{\circ} \mathrm{N}$ and $5^{\circ} \mathrm{S}$, slightly poleward of the amplitude peaks of the $n=1$ theoretical mode for a resting ocean, but near the latitude of the peak amplitude estimated both observationally and theoretically by Chelton et al. (2003) for a different wavenumber-frequency band. It should be noted, though, that the error bars in this analysis are large enough that the latitude of peak amplitude is subject to considerable uncertainty. The mode is essentially in phase at all latitudes, consistent with expectations for a free $n=1$ Rossby wave.

Because there is a large body of literature on the tropical instability wave, it is worthwhile to also examine the meridional structure of variability in the TIW band. In the $12.4^{\circ}$ wavelength, 31.5-day period band, a point within the TIW spectral peak, there is SSH variability coherent with that at $5.25^{\circ} \mathrm{N}$ at all latitudes between $9.75^{\circ} \mathrm{S}$ and $9.75^{\circ} \mathrm{N}$. The peak amplitude occurs at $5.25^{\circ} \mathrm{N}$ and the peak amplitude south of the equator $\left(4.75^{\circ} \mathrm{S}\right)$ is $3-5$ times smaller (Fig. $8 b$ ). There is a significant latitudinal phase variation, with about a $90^{\circ}$ phase shift between SSH variations at the nearequatorial latitudes and those near $8^{\circ} \mathrm{N}$ and $8^{\circ} \mathrm{S}$ (Fig. $8 \mathrm{e})$.

A theoretical prediction of the amplitude and phase of TIW SSH variability has been offered by Lyman et al. (2005), who used the zonal currents from a general circulation model to perform a linearized stability analysis by projecting the linearized equations of motion onto a set of baroclinic modes. There is a remarkable similarity of the observed amplitude and phase of TIW SSH variability in the $12.4^{\circ}$ wavelength, 31.5 -day period band to the amplitude and phase of SSH predicted by Lyman et al. (2005; their Fig. 11) for the $12.4^{\circ}$ wavelength, 31-day period band (Fig. 8). The model of Lyman et al. predicts a sea level phase difference of approximately $30^{\circ}$ between the equator and $2^{\circ} \mathrm{N}$ and $2^{\circ} \mathrm{S}$; this feature is present in the estimated meridional phase profile, but it is not statistically significant. However, if the meridional profiles are estimated using the equator as a reference latitude (not shown), the feature 

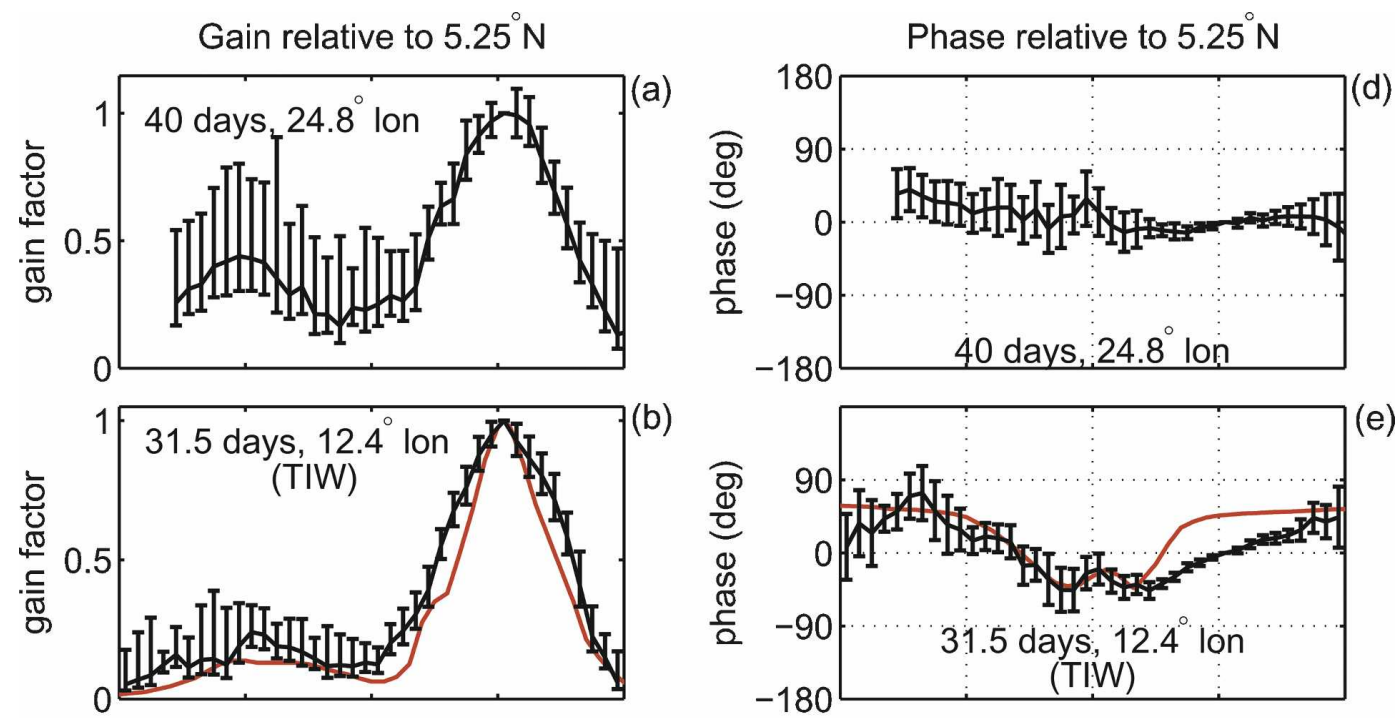

(b)
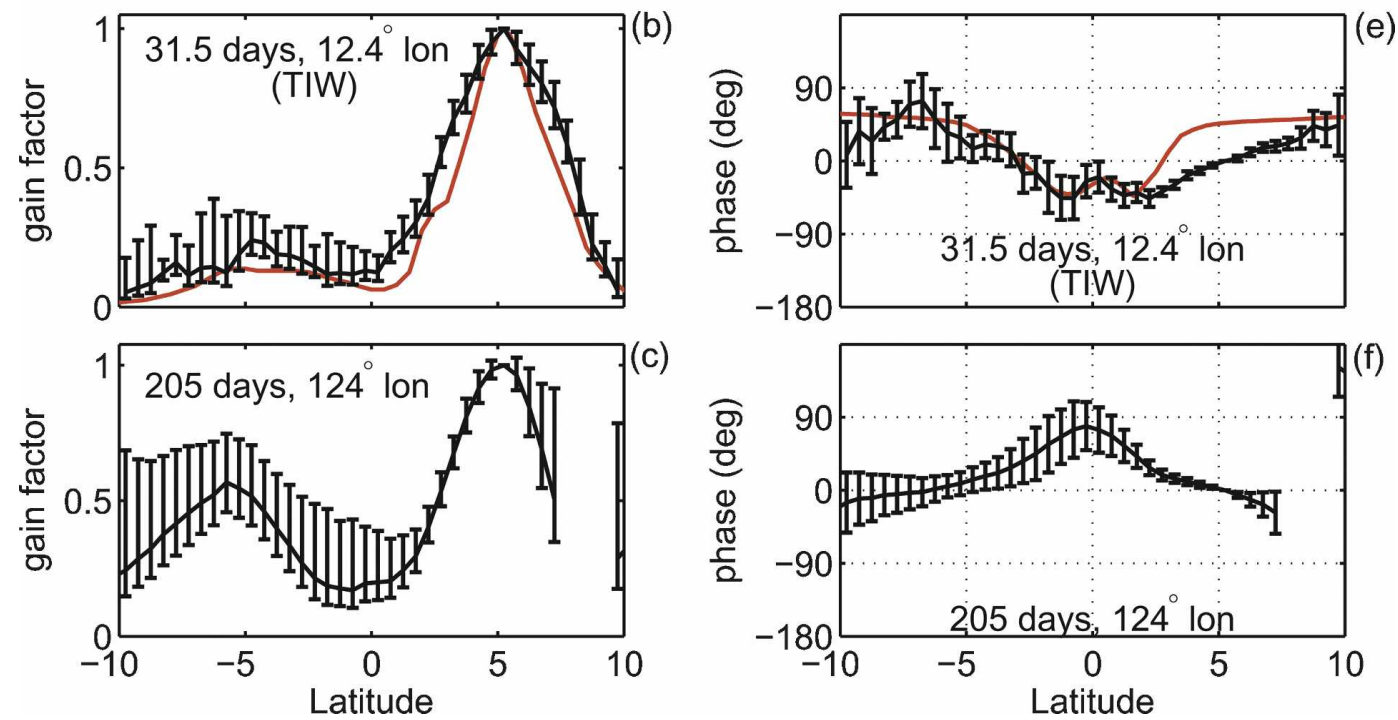

FIG. 8. Meridional profiles of SSH (a)-(c) amplitude and (d)-(f) phase relative to $5.25^{\circ} \mathrm{N}$ for points on the $n=$ 1 Rossby wave dispersion curve in Fig. 2. Periods and wavelengths at the central frequency and wavenumber of each band are indicated in each panel. In (b) and (e), the predictions of a linearized model (Lyman et al. 2005) for the same wavelength and period $\left(12.4^{\circ}, 31\right.$ days $)$ are also shown as red lines. As discussed in the text, the meridional structure in the low-wavenumber, low-frequency band [(c) and (f)] cannot be unambiguously ascribed to the $n=$ 1 Rossby wave.

is reproduced and, because the coherence with points near the equator is higher, the error bars are smaller and the subtle near-equatorial phase variations predicted by Lyman et al. are statistically significant. Also in common with the theoretical prediction is the observed $\sim 90^{\circ}$ phase shift between SSH variations at the near-equatorial latitudes and those near $8^{\circ} \mathrm{N}$ and $8^{\circ} \mathrm{S}$, with $\mathrm{SSH}$ variations at the equator lagging those at offequatorial latitudes. In the analysis of Lyman et al., this phase shift is a result of barotropic energy conversion associated with an equatorward eddy flux of zonal momentum. There is a significant difference between the predicted and observed phase relative to the equator over $2.75^{\circ}-8.25^{\circ} \mathrm{N}$, but the overall similarity between the observed and predicted structures provides independent evidence that the linearized model of Lyman et al. (2005) has captured much of the essential physics of the instability. The dispersion relation predicted by Lyman et al. is also in good agreement with the ob- served distribution of power in the broader TIW wavenumber-frequency band (pink line in Fig. 1).

Although the spectral resolution is too coarse to distinguish between the $n=1$ and higher-mode Rossby waves at very low frequencies and wavenumbers, it is instructive to examine the meridional structure of SSH for low-frequency, low-wavenumber, westward propagating variability. Such variability was considered by Chelton et al. (2003), who computed EOFs of spacetime filtered SSH with a goal of examining the meridional structure of basin-scale, annual-period Rossby waves. Because of the particular choice of frequencyband averaging made here, the meridional structure at the annual period cannot be resolved. Furthermore, the width of the Pacific basin places a fundamental restriction on the wavenumber resolution. Thus, for a qualitative comparison to the meridional structure inferred by Chelton et al. for basin-scale, annual-period variability, we consider the variability in the wavenumber- 
frequency band centered at a period of 205 days and a wavelength of $124^{\circ}$ longitude and encompassing periods of 153-312 days and wavelengths of $83^{\circ}-248^{\circ}$ (see the low-wavenumber, low-frequency box on Fig. 2).

The meridional structure estimated here for this lowfrequency, low-wavenumber variability shares some qualitative similarities with the meridional structure inferred by Chelton et al. (2003). The meridional profile of SSH amplitude resembles that expected for the first meridional mode, but the peak amplitudes are shifted slightly poleward (to about $5^{\circ} \mathrm{N}$ and $6^{\circ} \mathrm{S}$ ) compared to the prediction obtained from a model linearized about a state of rest. The amplitude of the peak in the Northern Hemisphere is larger than that in the Southern Hemisphere; here it is $1.75 \pm 0.42$ times larger, and it was also about 2 times larger in Chelton et al. (2003). (At 95\% confidence, the amplitude profile here is consistent with the ones inferred by Chelton et al.) As in Chelton et al., the Northern Hemisphere peak is sharper than the Southern Hemisphere peak. Finally, the meridional phase relationship also exhibits similarities to that inferred by Chelton et al. by a complex EOF analysis of filtered SSH data; both estimates show a tendency for off-equatorial variability to lag variability at the equator [Fig. 8 of this study; Fig. 9 of Chelton et al. (2003)].

It may be noteworthy that the Chelton et al. (2003) analysis, which allows examination of the meridional amplitude and phase structure as a function of longitude, shows considerable zonal variability in the meridional structure. Zonal variations in the amplitude and phase of the modal structures may be a factor contributing to the size of the $95 \%$ confidence intervals for the estimated modal structures. The confidence limits for the phase and gain estimates are dependent on the coherence amplitude, and zonal variation in the modal structures is one of a number of physical factors that may degrade the coherence between SSH signals at different latitudes and increase the uncertainty in the gain and phase estimates.

While it is tempting to conclude that the lowfrequency, low-wavenumber variability depicted in Figs. $8 \mathrm{c}$ and $8 \mathrm{f}$ is associated with the first meridionalmode Rossby wave, such a conclusion does not seem justified. More than one wave probably exists within the wavenumber-frequency band examined. For example, in the classical theory, there are three first baroclinic-mode dispersion curves that could contribute to variability in this wavenumber-frequency band, and the $n=1$ meridional mode of the second baroclinic mode could also contribute (see box marking this band in Fig. 2 ). These waves cannot be resolved from one another in this analysis and, in general, waves with similar fre- quencies and wavelengths of basin scale or larger will be difficult to resolve because the basin is too small for the relative phase of these waves to change significantly as they traverse the basin. Thus, we can only conclude that the meridional structure inferred for the 153-312day period, basin-scale wavelength band shares some similarities with the basin-scale, annual-period waves examined by Chelton et al. (2003) using another method, but the extent to which the meridional profile of variability in this band represents a particular type of wave is unclear. While this reservation about interpretation of basin-scale variability applies to some extent to the observational inferences of Chelton et al., their results are bolstered by good agreement of the inferred meridional structures with theoretical structures modified by interaction with the mean zonal currents.

\section{c. The $n=2$ Rossby waves}

There are few, if any, published accounts of the observed meridional structure of second meridional-mode Rossby waves in the ocean or atmosphere. Thus, it is of particular interest to examine the meridional structure of the variability leading to the isolated, well-defined, and statistically significant ridge of power in SSH near the $n=2$ dispersion curve. That the meridional structure inferred for the Kelvin and $n=1$ Rossby waves is consistent with theory and previous observations gives increased confidence that the spectral method gives sensible and useful results. For examination of the meridional structure of variability near the $n=2$ dispersion curve, the coherence amplitude, gain, and phase were estimated against $5.25^{\circ} \mathrm{S}$ for each latitude.

In the 42.9-50.1-day period, $14.6^{\circ}-16.5^{\circ}$ wavelength band, the meridional structure of the variability contributing to the spectral peak near the $n=2$ dispersion curve resembles expectations for an $n=2$ Rossby wave (Figs. 9a and 9c): the peak amplitudes in the Northern and Southern Hemispheres occur slightly poleward $\left(6^{\circ}\right.$ or $7^{\circ} \mathrm{N}$ and $\mathrm{S}$ ) of the peaks for the $n=1$ Rossby wave, and the structure is roughly antisymmetric in the sense that varibility is out of phase on opposite sides of the equator. That no coherent variability was detected near the equator is also consistent with expectations based on the zero mean-flow linear theory, which predicts a very small (less than $5 \%$ of the peak) amplitude for the $n=2$ mode near the equator. The phase between SSH variability near $5^{\circ} \mathrm{N}$ and $5^{\circ} \mathrm{S}$ is indistinguishable from $180^{\circ}$ (Fig. 9c), though, as for some of the Kelvin and $n=1$ Rossby waves, there is a phase drift moving poleward. In this case, the phase at $8.75^{\circ} \mathrm{S}$ leads that at $5.25^{\circ} \mathrm{S}$ by about $90^{\circ}$; there is a similar phase shift between $5.25^{\circ}$ and $9.75^{\circ} \mathrm{N}$ (and an almost equal phase shift between $5.25^{\circ}$ and $10.25^{\circ} \mathrm{N}$, not shown). 
Gain relative to $5.25^{\circ} \mathrm{S}$
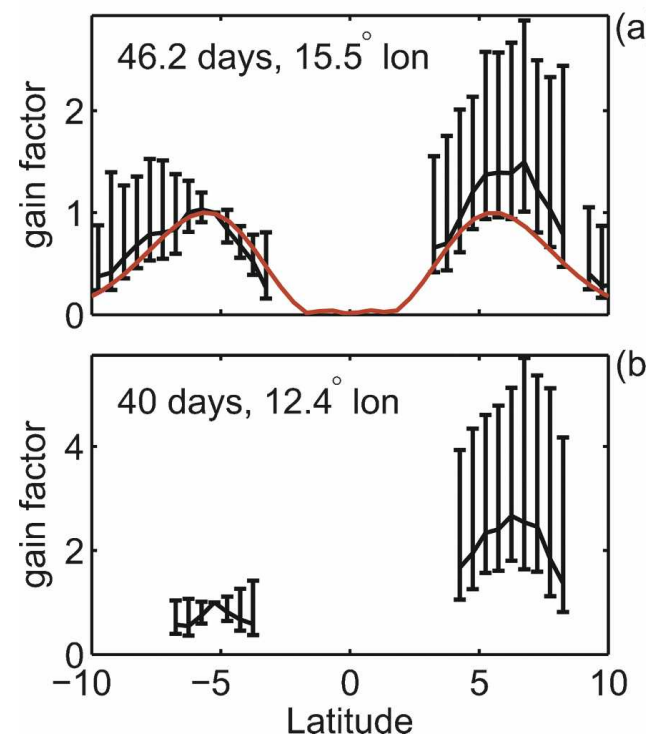

(a)

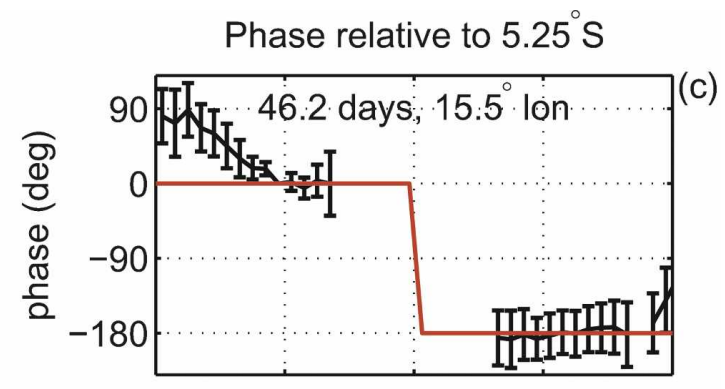

(b)

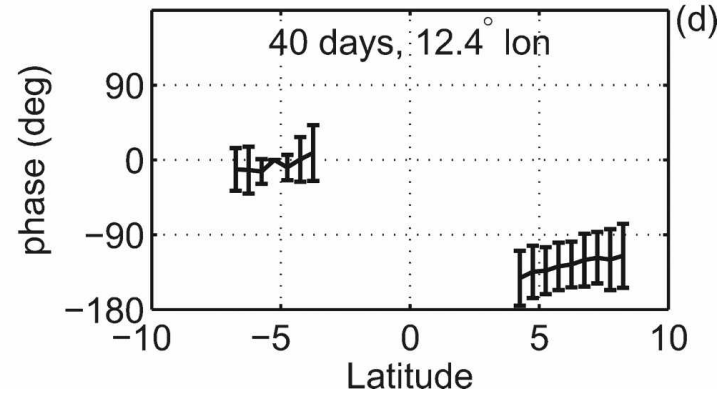

FIG. 9. Meridional profiles of SSH (c), (d) amplitude and (a), (b) phase relative to $5.25^{\circ} \mathrm{S}$ for points near the $n=2$ Rossby wave dispersion curve in Fig. 2. Periods and wavelengths at the central frequency and wavenumber of each band are indicated in each panel. For comparison, the red lines show the theoretical structure of the $n=$ 2 wave in a resting ocean. As expected of the $n=2$ mode, the peak amplitude in each hemisphere is shifted poleward with respect to that of the $n=1$ mode, and the variability is nearly $180^{\circ}$ out of phase on opposite sides of the equator.

In the 37.5-42.9-day period, $11.8^{\circ}-13.1^{\circ}$ wavelength band, the meridional structure of the variability contributing to the spectral peak near the $n=2$ dispersion curve also resembles that of the theoretical $n=2$ mode (Figs. 9b and 9d). However, there is a pronounced and statistically significant difference in the amplitude on opposite sides of the equator: the amplitude in the Northern Hemisphere is about 2.5 times larger than that in the Southern Hemisphere. Also, variability on opposite sides of the equator is not perfectly out of phase. Instead, the phase difference is roughly $135^{\circ}$. The meridional structure in the adjacent wavenumber bands on either side of this wavenumber-frequency band is very similar (not shown); the phase profiles are almost identical, and the amplitude profiles are similar but with a smaller difference between amplitude in the Northern and Southern Hemispheres.

One is led to speculate that there may be some analogy between the structure of this 40 -day, $12.4^{\circ}$ mode and the TIW mode (Fig. 8) in which the asymmetry and more complicated phase structure (neither in phase nor out of phase) are indicative of instability (Lyman et al. 2005). In many studies of TIWs (e.g., McPhaden 1996), the frequency of this 40-day mode has not been resolved away from the dominant frequency of TIWs, but this mode of variability seems to be distinct from variability at higher frequencies, as is evidenced by the spectral gap at this wavenumber (Fig. 2). (Evidence of a spectral gap at TIW wavenumbers is not entirely unambiguous, though since there is no significant gap in the next lowest wavenumber band corresponding to a wavelength of $13.8^{\circ}$ longitude.) The period of tropical instability waves is often estimated to be 20-40 days (e.g., Qiao and Weisberg 1995), but recent work indicates that there are distinct instabilities at 33- and 17day periods involving, respectively, unstable Rossby and mixed Rossby-gravity waves (Lyman et al. 2007). Perhaps this 40-day mode, exhibiting the highest power near the $n=2$ theoretical dispersion curve and a somewhat complicated meridional phase and amplitude structure, is associated with another distinct instability. In this context, it is worth noting that three distinct types of instabilities have been identified near $155^{\circ} \mathrm{W}$ by diagnosis of energy conversion terms in CTD and velocity observations (Luther and Johnson 1990).

\section{Conclusions}

An estimate of the distribution in wavenumber and frequency of sea level energy in the equatorial Pacific Ocean was presented. The approach used here allowed isolation of an equatorial Kelvin wave and two Rossby wave modes. A gross assessment of the extent to which variations in sea level are symmetric or antisymmetric 
was also presented. It was found that the symmetric spectrum exhibits a spectral peak near the $n=1$ Rossby wave dispersion curve for the first baroclinic mode, while the antisymmetric spectrum exhibits a spectral peak near the $n=2$ Rossby wave dispersion curve for the first baroclinic mode. Both of these results might be expected from classical equatorial wave theory (e.g., Matsuno 1966). Although the Kelvin wave and $n=1$ Rossby wave have been observed from a variety of perspectives in previous studies, the $n=2$ Rossby wave and its meridional structure have not been clearly isolated in the past; isolation of this mode is the principal result of this paper.

The meridional sea level structures associated with selected wavenumber-frequency bands were examined in more detail by extracting variability that was coherent with that at some reference latitude. The amplitude profile of intraseasonal Kelvin waves is well described (within the uncertainty in the analysis) by a Gaussian within about $5^{\circ}$ of the equator, but there is variability in sea level as far away as $11.75^{\circ} \mathrm{S}$ that is coherent with and leads Kelvin wave variability at the equator. This result is statistically robust, and this coherent offequatorial variability may ultimately be explained as being directly forced by the same intraseasonal winds that force the Kelvin waves. The meridional profiles of 40-day and semiannual (i.e., 153-312 day) Rossby waves near the theoretical $n=1$ dispersion curve were shown to resemble those identified in a previous analysis of annual-period Rossby waves (Chelton et al. 2003), with peak amplitudes being about two times larger in the Northern Hemisphere than in the Southern Hemisphere. In the tropical instability wave band, the meridional sea level structure showed remarkable similarities to the theoretical prediction made by Lyman et al. (2005) using a multiple baroclinic-mode model linearized about a realistic latitude-dependent and depthdependent zonal current system.

Two examples of the meridional structure of variability at wavenumbers and frequencies near the theoretical $n=2$ Rossby wave dispersion curve were presented, and these profiles grossly match expectations for first baroclinic-mode, $n=2$, Rossby waves. The variability is roughly antisymmetric, with variability on opposite sides of the equator being nearly $180^{\circ}$ out of phase. (However, one of the profiles and others not shown had phase angles closer to $135^{\circ}$ at opposite sides of the equator.) While there is considerable uncertainty in the meridional profiles of amplitude, the peak amplitude in each hemisphere is found slightly poleward of that for the $n=1$ mode, in accordance with theoretical expectations (e.g., Matsuno 1966). Like the $n=1$ Rossby waves, the amplitude of these waves is larger in the
Northern Hemisphere than in the Southern Hemisphere. It seems likely that this interhemispheric difference in amplitude is due to effects of the equatorial current system, in analogy to the effects on the $n=1$ mode.

Given only the sea level observations used in this paper, it is difficult to distinguish between the various vertical baroclinic modes. Previous observations of equatorial Kelvin and Rossby waves in subsurface data have found vertical phase propagation (e.g., Lukas and Firing 1985; Kessler and McCreary 1993; Kutsuwada and McPhaden 2002), a clear indication that this variability cannot be understood as a single vertical mode. A more complete interpretation of the observations presented here will require consideration of the vertical structure and propagation of these equatorial waves. Use of the subsurface observations from the TAOTRITON mooring array in the Pacific might allow some progress on this issue, but the fact that the TAOTRITON array normally does not measure temperature below 500-m depth is likely to lead to some ambiguities in distinguishing among various baroclinic modes.

Complications with interpretation of baroclinic modes in the TAO-TRITON mooring array notwithstanding, it would be interesting to see an analysis similar to this one performed using data from the TAOTRITON mooring array. Though the spectral calculation would be more severely band limited in wavenumber (nominally resolving variability at zonal wavelengths of $30^{\circ}-110^{\circ}$ of longitude), the good temporal resolution would allow examination of equatorially trapped gravity waves and mixed Rossby-gravity waves. Combined with the results presented here, analysis of data from the TAO -TRITON mooring array could thus allow mapping of nearly the entire zonal wavenumber-frequency dispersion space relevant to low vertical-mode equatorial waves, which would lead to a better understanding of the dynamics of transient motions in the equatorial region.

Acknowledgments. The author is grateful to the many scientists and engineers who carried out the TOPEX/Poseidon mission and to the WOCE program and NASA's Jet Propulsion Laboratory for providing the corrected and gridded sea level anomaly data. The author thanks Carl Wunsch and Ted Durland for helpful comments and discussion. Comments on the manuscript by Geoffrey (Jake) Gebbie, Bruce Warren, Billy Kessler, and Dudley Chelton are appreciated. The author also thanks the attendees of the WHOI Department of Physical Oceanography weekly discussion group for their feedback and encouraging comments on early results of this research. This work was supported 
in part by the NOAA Climate Prediction Program for the Americas (Grant NA17RJ1223).

\section{REFERENCES}

Bendat, J. S., and A. G. Piersol, 1986: Random Data: Analysis and Measurement Procedures. 2nd ed. Wiley, 566 pp.

Blandford, R., 1966: Mixed gravity-Rossby waves in the ocean. Deep-Sea Res., 13, 941-961.

Boulanger, J.-P., and C. Menkes, 1995: Propagation and reflection of long equatorial waves in the Pacific Ocean during the 1992-1993 El Niño. J. Geophys. Res., 100, 25 041-25 059.

$\longrightarrow$, and 1999: Long equatorial wave reflection in the $\mathrm{Pa}-$ cific Ocean from TOPEX/POSEIDON data during the 19921998 period. Climate Dyn., 15, 205-225.

Boyd, J., 1982a: The influence of meridional shear on planetary waves. Part I: Nonsingular wind profiles. J. Atmos. Sci., 39, 756-769.

_ 1982b: The influence of meridional shear on planetary waves. Part II: Critical latitudes. J. Atmos. Sci., 39, 770-790.

Brossier, F., 1987: Numerical modeling of equatorial waves in the presence of a mean current. J. Phys. Oceanogr., 17, 11001113.

Chelton, D., R. deSzoeke, M. Schlax, K. El Naggar, and N. Siwertz, 1998: Geographical variability of the first baroclinic Rossby radius of deformation. J. Phys. Oceanogr., 28, 433460.

— , F. Wentz, C. Gentemann, R. deSzoeke, and M. Schlax, 2000: Satellite microwave SST observations of transequatorial tropical instability waves. Geophys. Res. Lett., 27, 1239-1242.

—, M. Schlax, J. Lyman, and G. Johnson, 2003: Equatorially trapped Rossby waves in the presence of a meridionally sheared baroclinic flow in the Pacific Ocean. Prog. Oceanogr., 56, 323-380.

Cravatte, S., J. Picaut, and G. Eldin, 2003: Second and first baroclinic Kelvin modes in the equatorial Pacific at intraseasonal timescales. J. Geophys. Res., 108, 3266, doi:10.1029/ 2002JC001511.

Delcroix, T., J. Picaut, and G. Eldin, 1991: Equatorial Kelvin and Rossby waves evidenced in the Pacific Ocean through Geosat sea level and surface current anomaly. J. Geophys. Res., 96, 3249-3262.

_- J.-P. Boulanger, F. Masia, and C. Menkes, 1994: Geosatderived sea level and surface current anomalies in the equatorial Pacific during the 1986-1989 El Niño and La Niña. J. Geophys. Res., 99, 25 093-25 107.

Donohue, K., and M. Wimbush, 1998: Model results of flow instabilities in the tropical Pacific Ocean. J. Geophys. Res., 103, $21401-21412$.

Enfield, D., 1987: The intraseasonal oscillation in eastern Pacific sea levels: How is it forced? J. Phys. Oceanogr., 17, 18601876.

Eriksen, C., 1982: Equatorial wave vertical modes observed in a western Pacific island array. J. Phys. Oceanogr., 12, 12061227.

— ergy flux at 9- to 90-day periods in the central Pacific. $J$. Geophys. Res., 93, 15 455-15 466.

Farrar, J. T., and R. A. Weller, 2006: Intraseasonal variability near $10^{\circ} \mathrm{N}$ in the eastern tropical Pacific Ocean. J. Geophys. Res., 111, C05015, doi:10.1029/2005JC002989.

Hendon, H., and M. Salby, 1994: The life cycle of the MaddenJulian oscillation. J. Atmos. Sci., 51, 2225-2237.
, B. Liebmann, and J. Glick, 1998: Oceanic Kelvin waves and the Madden-Julian oscillation. J. Atmos. Sci., 55, 88-101.

Johnson, E., 1993: Effects of a mean three-dimensional flow on intraseasonal Kelvin waves in the equatorial Pacific Ocean. $J$. Geophys. Res., 98, 10 185-10 194.

—, and M. McPhaden, 1993: Structure of intraseasonal Kelvin waves in the equatorial Pacific Ocean. J. Phys. Oceanogr., 23, $608-625$.

Kessler, W., and J. McCreary, 1993: The annual wind-driven Rossby wave in the subthermocline equatorial Pacific. $J$. Phys. Oceanogr., 23, 1192-1207.

—, and M. McPhaden, 1995: Oceanic Kelvin waves and the 1991-93 El Niño. J. Climate, 8, 1757-1774.

— _ - and K. Weickmann, 1995: Forcing of intraseasonal Kelvin waves in the equatorial Pacific. J. Geophys. Res., 100, $10613-10631$.

Kutsuwada, K., and M. McPhaden, 2002: Intraseasonal variations in the upper equatorial Pacific Ocean prior to and during the 1997-98 El Niño. J. Phys. Oceanogr., 32, 1133-1149.

Lukas, R., and E. Firing, 1985: The annual Rossby wave in the central equatorial Pacific Ocean. J. Phys. Oceanogr., 15, 5567.

Luther, D., and E. Johnson, 1990: Eddy energetics in the upper equatorial Pacific during the Hawaii-to-Tahiti Shuttle Experiment. J. Phys. Oceanogr., 20, 913-944.

Lyman, J., D. Chelton, R. deSzoeke, and R. Samelson, 2005: Tropical instability waves as a resonance between equatorial Rossby waves. J. Phys. Oceanogr., 35, 232-254.

—, G. Johnson, and W. Kessler, 2007: Distinct 17-day and 33day tropical instability waves in subsurface observations. $J$. Phys. Oceanogr., 37, 855-872.

Matsuno, T., 1966: Quasi-geostrophic motions in the equatorial area. J. Meteor. Soc. Japan, 44, 25-43.

McPhaden, M., 1996: Monthly period oscillations in the Pacific North Equatorial Countercurrent. J. Geophys. Res., 101, 6337-6359.

_, and R. Knox, 1979: Equatorial Kelvin and inertio-gravity waves in a zonal shear flow. J. Phys. Oceanogr., 9, 263-277.

— , and B. Taft, 1988: Dynamics of seasonal and intraseasonal variability in the eastern equatorial Pacific. J. Phys. Oceanogr., 18, 1713-1732.

— , and P. Ripa, 1990: Wave-mean flow interactions in the equatorial ocean. Annu. Rev. Fluid Mech., 22, 167-205.

, J. Proehl, and L. Rothstein, 1986: The interaction of equatorial Kelvin waves with realistically sheared zonal currents. J. Phys. Oceanogr., 16, 1499-1515.

— (TOGA) observing system: A decade of progress. J. Geophys. Res., 103, 14 169-14 240.

Meyers, S., and J. O'Brien, 1994: Spatial and temporal 26-day SST variations in the equatorial Indian Ocean using wavelet analysis. Geophys. Res. Lett., 21, 777-780.

_ B. Belly, and J. O'Brien, 1993: An introduction to wavelet analysis in oceanography and meteorology: With application to the dispersion of Yanai waves. Mon. Wea. Rev., 121, 28582866.

Moore, D., and S. Philander, 1977: Modelling the tropical ocean circulation. The Sea-Ideas and Observations on Progress in the Study of the Seas, E. D. Goldberg, Ed., Marine Modeling, Vol. 6, John Wiley and Sons, 319-361.

Munk, W., and D. Moore, 1968: Is the Cromwell Current driven by equatorial Rossby waves? J. Fluid Mech., 33, 241-259.

Perez, R., D. Chelton, and R. Miller, 2005: The effects of wind 
forcing and background mean currents on the latitudinal structure of equatorial Rossby waves. J. Phys. Oceanogr., 35, 666-682.

Périgaud, C., 1990: Sea level oscillations observed with Geosat along the two shear fronts of the Pacific north equatorial countercurrent. J. Geophys. Res., 95, 7239-7248.

Polito, P. S., and O. T. Sato, 2003: Patterns of sea surface height and heat storage associated to intraseasonal Rossby waves in the tropics. J. Geophys. Res., 108, 3373, doi:10.1029/2002JC001684.

Qiao, L., and R. Weisberg, 1995: Tropical instability wave kinematics: Observations from the Tropical Instability Wave Experiment. J. Geophys. Res., 100, 8677-8693.

Ripa, P., and S. Hayes, 1981: Evidence for equatorial trapped waves at the Galápagos Islands. J. Geophys. Res., 86, 65096516.

Roundy, P., and W. Frank, 2004a: A climatology of waves in the equatorial region. J. Atmos. Sci., 61, 2105-2132.

_ and _ 2004b: Effects of low-frequency wave interactions on intraseasonal oscillations. J. Atmos. Sci., 61, 3025-3040.

- , and G. Kiladis, 2006: Observed relationships between oceanic Kelvin waves and atmospheric forcing. J. Climate, 19, 5253-5272.

Schlax, M., and D. Chelton, 1994: Aliased tidal errors in TOPEX/ POSEIDON sea surface height data. J. Geophys. Res., 99, $24761-24775$

Takayabu, Y., 1994: Large-scale cloud disturbances associated with equatorial waves. Part I: Spectral features of the cloud disturbances. J. Meteor. Soc. Japan, 72, 433-448.

Tsai, P., J. O'Brien, and M. Luther, 1992: The 26-day oscillation observed in the satellite sea surface temperature measurements in the equatorial western Indian Ocean. J. Geophys. Res., 97, 9605-9618.

Wakata, Y., 2007: Frequency-wavenumber spectra of equatorial waves detected from satellite altimeter data. J. Oceanogr., $\mathbf{6 3}$, 483-490.

Wallace, J., and V. Kousky, 1968: Observational evidence of Kelvin waves in the tropical stratosphere. J. Atmos. Sci., 25 , 900-907.

Wheeler, M., and G. Kiladis, 1999: Convectively coupled equatorial waves: Analysis of clouds and temperature in the wavenumber-frequency domain. J. Atmos. Sci., 56, 374-399.

Wunsch, C., and A. Gill, 1976: Observations of equatorially trapped waves in Pacific sea level variations. Deep-Sea Res., 23, 371-390.

Yanai, M., and M. Murakami, 1970: Spectrum analysis of symmetric and antisymmetric equatorial waves. J. Meteor. Soc. Japan, 48, 331-346.

Zang, X., L.-L. Fu, and C. Wunsch, 2002: Observed reflectivity of the western boundary of the equatorial Pacific Ocean. J. Geophys. Res., 107, 3150, doi:10.1029/2000JC000719.

Zangvil, A., and M. Yanai, 1980: Upper tropospheric waves in the tropics. Part I: Dynamical analysis in the wavenumberfrequency domain. J. Atmos. Sci., 37, 283-298. 